\title{
A Learning Process Which Improves Symmetry in the Dynamics of Thoracic Rotation: A New Hope for the Amelioration of Motor Disabilities
}

\author{
Vinicius Monteiro Diederichs* \\ John Cabot University, Rome, Italy \\ Email:info@monteirodiederichs.com
}

How to cite this paper: Diederichs, V. M. (2019). A Learning Process Which Improves Symmetry in the Dynamics of Thoracic Rotation: A New Hope for the Amelioration of Motor Disabilities. Advances in Physical Education, 9, 145-175.

https://doi.org/10.4236/ape.2019.93011

Received: April 24, 2019

Accepted: July 5, 2019

Published: July 8, 2019

Copyright $\odot 2019$ by author(s) and Scientific Research Publishing Inc. This work is licensed under the Creative Commons Attribution-NonCommercial International License (CC BY-NC 4.0). http://creativecommons.org/licenses/by-nc/4.0/

\begin{abstract}
Intersegmental joint dynamics permit generalisability: that is, a combination of joints to achieve maximum attainable amplitude of one degree of freedom. Generalisability contains the information to configure all lesser amplitudes using many degrees. This paper describes a treatment to reduce asymmetry in thoracic rotation, which appears to cause motor disabilities and pain. It proposes a learning process to recalibrate the neuromuscular system. The treatment is based on classical conditioning in which actors receive instructions to control a specific coordinate of the dominant hand-the conditioned stimulus (CS) - to be paired with a tensile force-the unconditioned stimulus (US). This pairing of CS with US generates a sequence of events, the conditioned response $(C R)$. To facilitate control, the hand first reaches the target position constraining the overall degrees of freedom to just one. This reduces the burden on the CNS to deal with the indeterminacy of limb lengths, the regulation of joint rotation and the combination of multiple joints for performing the motor task. The dynamics of this CR generates coupling, comparable to the dynamics described in coupling of posture and gait. To verify the theory: in Experiment 1, thirteen participants with acute motor impairment received three treatments; in Experiment 2, twenty-six healthy participants were randomly assigned into two groups to perform the experimental treatment with the dominant or the non-dominant hand, respectively, for comparison. Seven variables were measured: four ranges of motion, two perceived efforts, and one pain. In Groups 2 and 3, the improvement in thoracic symmetry was significant. The treatment is able to trigger a mechanism that detects a critical value and initiates a transition from the dynamics of the action system and task constraints to a default value. Additionally, the treatment is highlighted as a neuromodulation impacting muscle tone with long-lasting amelioration of motor disabilities and pain.
\end{abstract}




\section{Keywords}

Spherical Coordinate System, Size Scaling, Serial Reaction Time, Motor Sequence Learning, Limb Impedance, Neuromodulation, Muscle Tone, Gravitational Force

\section{Introduction}

Treatment of motor disabilities, based on a learning process, demonstrates efficiency when the lower limbs' impedance is augmented (Diederichs, 2018). One way to augment impedance is by using elastic tubes, its tensile force acting to pull the pelvis towards the feet (the base of support), which facilitates recalibration of the neuromuscular system. In this example, the top-to-bottom arrangement of elastic tubes applies a tensile force which stabilizes the pelvis. It also permits pelvic movement: a dynamic swaying of maximum amplitude to both sides, while hands and feet are held stationary (cf. Diederichs, 2018). New \& Noteworthy: There is evidence that elastic tubing can be used to simulate the vertical component of the ground reaction force. In natural coordination mode, such as walking at comfortable speed, the feet alternate between three phases. swing, heel strike and stance phases. Considering only the end of the stride cycle, the actor is confident that the heel will strike the ground for the upcoming stance phase. Normally, forward walking does not require conscious control of equilibrium. Fine equilibrium control is an elemental motor-behavior occurring especially when only one foot supports the entire body. Thus, when a heel strikes the ground, muscles must react synergically, using reflexes to maintain the body's centre of mass inside the boundaries of the base of support. The foot remains stationary on the ground supporting the contralateral side of the body for a dwell time, allowing the other foot to swing forward. Instead of using the feet, a tensile force can be controlled by use of the hands if both the kinematic configuration and orientation of the body are facilitated.

This paper describes an effective treatment for reducing asymmetries in thoracic rotation which appears to cause motor disabilities and pain (van Dillen et al., 2006; Seay et al., 2011; van den Hoorn et al., 2012; Tanigawa et al., 2018), using a learning process to recalibrate the neuromuscular system. This treatment is based on classical conditioning (Moore \& Choi, 1997), in which the actor receives the instruction to control a specific coordinate of the dominant hand-the conditioned stimulus (CS) - to be paired with a tensile force, the unconditioned stimulus (US). Combinations of joints to achieve the maximum reachable amplitude with only one degree of freedom (DOF) contain information to enable the configuration of all lesser amplitudes using many DOFs (Amazeen et al., 1998). Furthermore, the accuracy of movement varies with speed (Fitts, 1954): if a movement's force increases, the neural noise also increases (Schmidt et al., 1979), reducing the accuracy with which the location of a target is estimated. At 
this point, it becomes reasonable first to reach the target coordinate which is in the maximum reachable position, let us say in position $\mathrm{A}$, and only then pair the conditioned with the unconditioned stimulus. This reduces the burden on the central nervous system (CNS) of dealing with the indeterminacy of limb lengths, regulating joint rotation, and combining multiple joints for performing a motor task (Bernshteĭn, 1967).

Theoretically, asymmetric rotation of the thorax can also be caused by a dynamic dominance which results from a preference to use one body side to perform fine movements while the other, the non-dominant side, is used as a beam or stabiliser (Diederichs, 2017). This side dominance is believed to affect the conditioning state of oblique abdominal muscles (OAM) with an impact on motor control due to an unusual top-to-bottom asymmetry of the OAM. Asymmetry in thoracic rotation is due to malfunction of the muscle spindles in the side of the OAM that is less sensitive to tension, indicating an impairment in the stretch reflexes (cf. Diederichs, 2017). Additionally, many actions and tasks are executed from a sitting position, which highlights that the sedentary position is characterized by thoracic rotation while the pelvis remains stationary. This demonstrates that thoracic stability depends mostly on feedback from the muscle spindles, rather than from vision or the vestibular system, when the pelvis is stationary (van Drunen et al., 2016). Furthermore, in the experimental comparison of the left and right-side upper limbs, shoulders are equally able to control discrete movements which are important for performance of fine motor skills (Newell \& van Emmerik, 1989). But with the engagement of additional DOFs-from the elbow and wrist-the control of fine motor skills becomes dominant in only one side (Newell \& van Emmerik, 1989). This suggests that both the left and right-side shoulders are able to control one degree of freedom when the hand is in the maximum reachable amplitude, and thus facilitates learning transfer and generalisability (Aune et al., 2017).

Empirically, in this treatment the actor learns to stabilize the thorax while maintaining the hand in the maximum reachable amplitude, in position $\mathrm{A}$, while the tensile force challenges the specified coordinate. This tensile force is counteracted by the actor's dominant hand while the contralateral hand actively maintains the thorax stable. As an example, the pelvis is stabilized on specially designed equipment, allowing one hand to ensure stability of the thorax while maintaining the other hand in its maximum reachable amplitude (Figure 1). The final result after pairing CS with US is the conditioned response (CR), which generates a coupling comparable to the dynamics described in the theory for coupling of posture and gait (Kay \& Warren, 2001). However, the CR entails a dynamic rearrangement of all body segments, which is the motor rule. The motor goal is a specific coordinate, but due to the single DOF the hand suddenly drifts from the specified coordinate, causing disruption in the sequential regularity of the training phase. Training requires the actor to discriminate between the specific coordinate (s)he needs to maintain and the actual coordinate, 


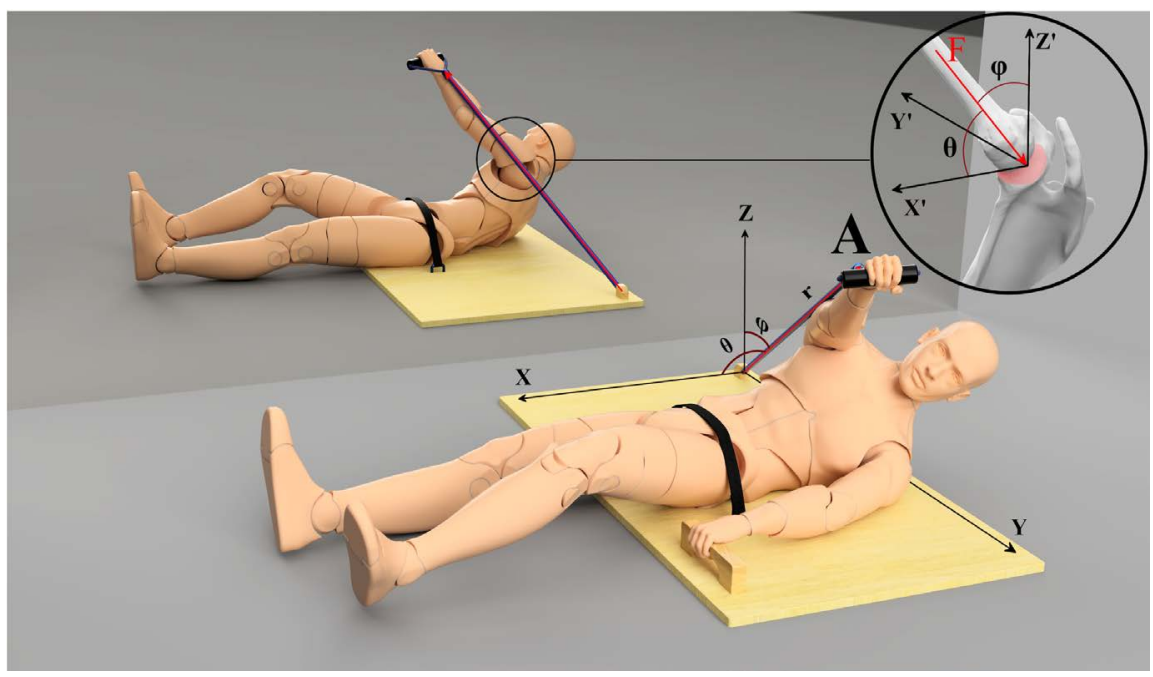

Figure 1. Illustrates the orientation and the final kinematic configuration after pairing CS with US, stationary for a dwell time in position A. The actor holds an elastic tubing which is under tension and has two points of action, one-point acts on the hand and the other is transferred to the ground (e.g. Kourtidou-Papadeli et al., 2008). This elastic tubing applies a tensile force in the direction of maximum variance, which depends on the dynamics of the action system and task constraints (Gritsenko et al., 2016). Note: while the right shoulder is stabilized in the vertical axis, the elastic tubing pulls in retroversion, which first makes the hand drift in a transverse plane in the same vertical axis. Better to understand, consider the elastic tubing as a radius of the positioning vector, which is defined by a spherical coordinate system. In this case, position A is described with these parameters: $r, \Theta$, and $\varphi$. We can assume that $r$ has a constant direction but variable magnitude which depends of the hand position. Variation in $r$ describes the change in the hand's position and tension force; variation in $\Theta$ and $\varphi$ describes the hand drifting from position A. Therefore, when the elastic tubing is transferred to the actor, (s)he counteracts the vertical force but the hand suddenly drifts, moving in the sagittal plane around a transversal axis as a result of the glenohumeral joint's degrees of freedom (Figure 3). After a reaction time, a discrete movement is generated, the hand moves back to the specified coordinate. However, by pairing CS with US the kinematic configuration of the lower limbs reacts naturally for controlling the equilibrium (Caillou et al., 2002). It is important to note that the shoulder is maintained stable in the vertical axis. Shoulder's stability is possible by the reaction of the contralateral OAM which maintains the thoracic transverse-position stabilized. It assumes that the glenoid fossa of the scapula is the origin of a point which is maintained stable by the reaction of the contralateral OAM (see sketch inside the circle). Here, the glenoid fossa of scapula is stabilized, but the shoulder musculature then has to modulate an effective force in order to avoid the hand drifting to the sides.

enabling incidental sequence learning (ISL) to occur, this is a characteristic of a serial reaction time (SRT) task (Rünger \& Frensch, 2008). Systematic, rather than random, transfer increases the availability of knowledge of the sequence of events as actors seek the cause of an experienced deviation from the expected task performance (Rünger \& Frensch, 2008).

In summary, instruction is delivered to use both hands with precisely the same level of effort. First, the actor's hand is moved passively towards position 
A, resulting in maximum torsion of the spine. At a signal, the actor maintains the hand at the maximum reachable amplitude, the elastic tubing is then transferred. Due to the glenohumeral joint, an unexpected DOF causes the hand to drift from the specified coordinate. Immediately after the reaction time (RT), a motor command is issued, and a discrete movement brings the hand back to position A, which is then maintained for a dwell time. After that dwell time, the actor receives instruction to release the arm and move the hand toward the shoulder while the nose is maintained stationary in position relative to the pelvis. In comparison with other approaches, the above approach has the advantage of being effective across a large range of motor disabilities. Further advantages include that the cost of implementation is low, and efficiency for motor rehabilitation is high. Indicating that adoption of this learning process in clinics and hospitals would prove a valid integrative medical approach.

\section{Methodology}

In order to compare the conditioning effect of this treatment, two different experiments were set up. In Experiment 1, a group of thirteen participants suffering specific, acute motor impairment (Group 1) was followed in three sessions, over a period of two up to three weeks. In Experiment 2, twenty-six healthy participants were randomly assigned to Groups 2 or 3, and performed the treatment with the dominant and the non-dominant hand, respectively. In Group 3, participants were informed that performing the treatment with the non-dominant hand would not improve their condition and used the non-dominant hand to eliminate a placebo effect. However, this study indicates that, in a passive muscle, the spindles' primary afferents fire in direct relationship to muscle force-related variables, rather than to length-related variables (Blum et al., 2017). The kinematics of the treatment described here is designed to permit a passive short-range stiffness of the OAM, made possible by using the ipsilateral hand to stabilize the thorax in order to maintain the OAM short and passive. This study hypothesizes that, whenever a population of muscle spindles from the OAM in one side only is correlated with asymmetry of thoracic rotation, treatment improves the dynamics of thoracic rotation by inducing passive short-range stiffness in the OAM in only one side. The following section expounds the procedure.

\subsection{Experimental Treatment}

After receiving preliminary information, the actor takes up a supine position, the pelvis then being stabilized by a belt. The CS was instructed through four stages as follows. First, information was provided on how to stabilize the thorax: "With one hand, grip the handle forcefully to maintain the thorax stable while, with the other, also exerting a strong grip to maintain position A. Both hands must perform at the same level of effort. It is important to control the stability of the thorax by maintaining a strong grip in both hands." Second, now with one hand al- 
ready stabilizing the thorax, the actor's preferred hand is passively moved towards its maximum amplitude. From position A, the actor is required to observe his hand, forearm, arm and shoulder. The clinician then continues: "Imagine a virtual line starting from the hand, in position $\mathrm{A}$, and extending to the shoulder, in position B". This reinforces the understanding that position $\mathrm{A}$ is at the maximum amplitude that can be attained. Third, the actor is now required to move the hand in a virtual straight line from position A to B while maintaining the nose's position stationary. For that, the clinician counteracts the movement by holding the actor's hand while keeping a thumb touching the actor's nose. In this rehearsal the actor learns how to stabilize the thorax while performing a discrete movement. Note that the direction of the force during this rehearsal is inverted considering the direction of the US tensile force.

In the fourth stage, with the actor resting in position, the clinician reinforces the CS. "Remember to use both hands with the same level of effort; while one hand gives stability to the thorax the other must be stationary in position A. Hold position A very firmly. This requires effort but takes no longer than two seconds. Once you have held position A for this short time, I give a signal to release your arm to move your hand towards position B. Pay attention to this spoken instruction: 'Three, two, one' which is the indication to alert you that the signal is imminent. The signal to start to counteract the tensile force is given the moment that I say 'Now!' If that is clear, what you must do is to keep your hand in position A for about two seconds. It is not so important you move towards position B. But if you change your mind and decide to drop out, you need only to release your arm." Once the actor indicates being confident with the instructions, the actor's hand is passively moved towards position A, then the clinician asks: "Are you ready?" Receiving confirmation, the clinician prompts: "Three, two, one, Now! Hold the position". The tensile force, i.e. the US, is then transferred to the actor's hand. The actor efficiently anticipates the reaction to counteract the tensile force, i.e. the $\mathrm{CR}$, maintaining the maximum reachable amplitude. But the hand suddenly drifts from the specified coordinate, and then after a reaction time, a discrete movement returns the hand back to the specified coordinate for a dwell time. Then instruction is given to release the arm in order to move the hand towards position B while maintaining the nose position stationary. The procedure-i.e., the stimulus-is repeated five times.

\subsection{Preliminary Information and ROM Test}

This information highlights the importance of controlling the quality of range of motion (ROM), as naïve actors can be concerned by unexpected delayed-onset muscle soreness (DOMS). In this example ROM was checked by performing a squatting test, or other test elaborated ad hoc for each specific motor impediment (e.g., Holt et al., 2010). Thus, the neck rotation to a certain angle is impeded, the actor scores the level of pain for that specific degree of rotation. The same is done for other compartments, that is, shoulder, elbow, lumbar spine and so on. Actors suffering an overt acute motor impairment also performed this 
scoring test (Experiment 1). Once the specific test was clearly understood by the actor, information about the probability of developing DOMS after treatment was given. The actor is reassured that a better ROM signifies an improvement in their condition. Thus, even when DOMS causes a sensation of stiffness, joint ROM must be verified. "This is a normal change in muscle tone. When a natural change in muscle tone occurs, it is positive and important for improving the fitness threshold by strengthening of the muscles, especially in the lower back. This DOMS will probably begin one to seven days following treatment. You must remember to check ROM. Even if your muscles are sore and you are feeling stiffness, a better ROM means that muscle tone is changing for the good. Thus, there is nothing to cause you concern."

\subsection{Participants}

A total of thirty-nine right-handed participants (from office workers to construction workers), of both genders, exhibiting asymmetry in passive rotation of the shoulder girdle (assessed when sitting), engaged in the two experiments. The sample size was calculated by power analysis, and a larger sample was chosen as convenient. The raw dataset, criteria used to select right-handed participants, description of their initial condition and procedure adopted to score perceived effort are available in the Appendix. After the preliminary assessment, in all groups participants were allocated randomly, including in Group 1; that is, independently of the body's compartment affected by motor impairment. Experiment 1 involved thirteen participants suffering an acute, painful motor impairment. Experiment 2, twenty-six healthy participants able to perform motor tasks normally. All participants provided written informed consent to participate in the experiment, following a full explanation of procedures pre-approved by the local ethics committee. Much of the experiment was performed in the facilities of the University of Rome-Foro Italico, other sessions at John Cabot University, Rome. In Experiment 1 the participants (Group 1; 7 male, average age $44 \pm 10$ years, height $173 \pm 8 \mathrm{~cm}$, weight $71 \pm 10 \mathrm{~kg}$ [mean \pm standard deviation]) received a total of three treatments over different days, lasting two up to three weeks. In Experiment 2, participants were randomly assigned to Group 2 (10 male and 3 female, age $39 \pm 11$ years, height $178 \pm 11 \mathrm{~cm}$, weight $77 \pm 16 \mathrm{~kg}$ ) and Group 3 (10 male and 3 female, age $36 \pm 10$ years, height $176 \pm 9 \mathrm{~cm}$, weight $77 \pm$ $11 \mathrm{~kg}$ ). None of the participants dropped out during the procedure. The experiments were performed from the beginning of May until the end of July 2018. Data on seven different variables were collected before and after treatment. The procedure was recorded on video ( $4 \mathrm{k}$ from $100 \mathrm{~cm}$ distance) to analyze the time between CS and US, i.e. the interstimulus interval (ISI), by a person naïve to the study (see Appendix, Note 5).

\subsection{Apparatus}

A specially designed piece of equipment was used to ensure stability of the pelvis and provides handles for gripping. Elastic tubing was hooked onto the equip- 
ment; this was provided with a cushioned handle, ensuring a comfortable grip, and a safety cable. The resultant tensile force-magnitude is $250 \mathrm{~N}$ when the elastic tubing is extended to $125 \mathrm{~cm}$ (further information about the equipment is available on request). In Experiment 1, pain and effort were assessed using a numeric rating scale. In Groups 2 and 3, perceived effort was measured with a Rated Perceived Exertion (RPE)scale (Borg, 1982) and pain with a Category Ratio (CR10) scale (Borg, 1998). ROM of joints was measured with a dual digital inclinometer, Accumar model ACU002.

\subsection{Notes on Variables}

In order to test the hypothesis, seven different variables were measured: four were ROM-related; two were self-reported perceived effort; while one was pain-related motor impairment scored in Group 1 or pain-related movement scored in Groups 2 and 3. The full seven variables, and the procedure for data collection to assess the treatment, are as follows:

$V 1$ neck maximum passive flexion. The degree of inclination of the first thoracic vertebra and top of the skull was measured from a sitting position: the actor's hands resting supine above the knees while elbows are bent and resting above the thighs. First, the maximum passive flexion of both the lumbosacral and thoracic spine was established by gently guiding the actor into position; then the maximum passive flexion of the neck by asking the actor to release the head downward (see Appendix, Note 4).

$V 2$ thoracic maximum passive flexion. The degree of inclination of the first and the last thoracic vertebrae were measured from a standing position: the actor's hand was supine resting on a support. While the actor's knees remained locked, the clinician gently guided the pelvis to move slightly forward and the neck to its maximum passive flexion, in order to measure the maximum passive flexion of the thorax (see Appendix, Note 4).

$V 3$ thoracic maximum active rotation, right side up. The degree of rotation of the first thoracic vertebra was measured from a quasi-crawling position: the actor sat on the heels with knees flexed, the left elbow touching the ipsilateral knee while the right hand held the nape of the neck. The pelvis was stabilized in place by the clinician (see Appendix, Note 4 and Figure 4).

$V 4$ thoracic maximum active rotation, left side up. The degree of rotation of the first thoracic vertebra was measured from a quasi-crawling position: the actor sat on the heels with knees flexed, the right elbow touching the ipsilateral knee while the left hand held the nape of the neck. The pelvis was stabilized in place by the clinician (see Appendix, Note 4 and Figure 4).

$V 5$ isometric contraction, right side up. Using a Borg scale from 6 to 20 for Groups 2 and 3 (and a scale from 1 to 10 for Group 1). Perceived effort was scored from a quasi-crawling position: the left elbow rested on the equipment perpendicular to the ipsilateral shoulder, the right hand rested on the equipment perpendicular to the ipsilateral shoulder; the knees perpendicular to the hips and 
the feet flexed. To begin, actors were asked to lift slightly both knees together and then rotate towards the ceiling approaching the pelvis on the equipment's surface. This position was then held for fifteen seconds (see Appendix, Note 4 and Figure 4).

V6 isometric contraction, left side up. Using a Borg scale from 6 to 20 for Groups 2 and 3 (and a scale from 1 to 10 for Group 1), perceived effort was scored from a quasi-crawling position: the right side elbow rested on the equipment perpendicular to its ipsilateral shoulder, the left hand rested on the equipment perpendicular to the ipsilateral shoulder, the knees perpendicular to the hips and the feet flexed. To begin, actors were asked to slightly lift both knees together and then rotate towards the ceiling approaching the pelvis on the equipment's surface. This position was then held for fifteen seconds (see Appendix, Note 4 and Figure 4).

$V 7$ pain. The level of pain was scored on a numeric scale from 1 to 10, in Experiment 1. Although Groups 2 and 3 were characterized by a lack of impairment, pain was scored after a movement-screening test, which measured the difference between right and left sides of the body for each specific compartment, in a Borg scale from 0 to 10 (see Appendix, Note 3).

\subsection{Statistical Analysis}

To reduce bias during data collection, tests-especially for $V \mathbf{3}$ and $\mathbf{V} \mathbf{4}-$ were repeated up to three times. The difference in scales, between Experiments 1 and 2, for variables $V \mathbf{5}, V \mathbf{6}$ and $V \mathbf{7}$ were normalized. Analysis of regressions and mixed effects (linear mixed-effects models (Bates et al., 2015), using the lmer package in R) was used to calculate whether a predictor variable is significant or not. The significance of the difference with the mean was calculated with a post-hoc $t$-test (degrees of freedom based on Satterthwaite's lmer Test package in $\mathrm{R})$. The asymmetry in rotation of the thorax was calculated as the difference between $V 3$ and V4. In the Results section, the effect of the treatment for improving the thoracic symmetry is given for Groups 2 and 3. For the statistical analysis, the data of two participants (3B and 5C: the data set is available in Appendix) were excluded due to a remaining asymmetry after treatment. In Group 1, it was not possible to analyse the asymmetry due to the non-homogeneity of the sample; classification of the impairment (according to the World Health Organisation's guideline) is given in the Appendix (Note 3). The results are summarised and discussed below.

\section{Results}

Variable $V 1$ in Group 1, i.e., the passive flexion of the neck was significantly different in the first section $(t=-4.23, \mathrm{df}=62, p<0.001)$, but the difference between sessions 2 and 3 was not significant; $V 1$ in Groups 2 and 3 (there was no difference between Groups), the before and after was significant $(t=-2.35, \mathrm{df}=$ $25, p=0.027)$. Variable $V 2$ in Group 1, i.e., the thoracic passive flexion was sig- 
nificantly different $(t=-2.04, \mathrm{df}=62, p=0.046)$, as were the differences between sessions 1 and $2(t=-2.58, \mathrm{df}=62, p=0.012)$, and between sessions 2 and $3(t=-2.36, \mathrm{df}=62, p=0.022) ; V 2$ was not significant in Groups 2 and 3 . Of interest, variable $V 3$ was not significant for Groups 1, 2 and 3. Asymmetrically, variable $V 4$ was significantly different in Group $1(t=-2.63, \mathrm{df}=62, p=$ $0.011)$, and also between sessions 1 and $3(t=-2.42, \mathrm{df}=62, p=0.019) ; V 4$ was significant also in Groups 2 and $3(t=-3.11, \mathrm{df}=25, p<0.01)$. Variable $V 5$ in Group 1, i.e., a change in the sensation of effort, was significantly different $(t=$ $3.79, \mathrm{df}=62, p=0.001)$; the differences were significant between sessions 1 and $3(t=4.97, \mathrm{df}=62, p<0.001)$, and sessions 2 and $3(t=3.53, \mathrm{df}=62, p<0.001)$; $V 5$ was also significantly different in Groups 2 and $3(t=2.63$, df $=25, p=$ $0.014)$. Variable $V 6$ in Group 1 was significantly different $(t=2.84, \mathrm{df}=62, p<$ $0.001)$; the difference was significant between sessions 1 and $2(t=3.29, \mathrm{df}=62$, $p<0.01$ ). In Group 2, but not in Group 3, the difference in $V 6$ was significant ( $t=$ $3.63, \mathrm{df}=24, p<0.001$ ). The amelioration of pain (i.e. variable $V 7$ ) after the treatment was significantly different in Group $1(t=6.91, \mathrm{df}=62, p<0.001)$; the differences were significant between session 1 and $2(t=6.67, \mathrm{df}=62, p<0.001)$ and between session 1 and $3(t=8.21, \mathrm{df}=62, p<0.001)$. In Groups 2 and 3 , the difference in $V 7$ was significant $(t=6.33$, df $=25, p<0.001)$. When testing for symmetry in Groups 2 and 3, the improvement in the symmetry of thorax rotation was significant $(t=-7.05, \mathrm{df}=18, p<0.01)$.

\section{Discussion}

The major intention of the present study is to describe an effective and efficient approach to improve symmetry in the dynamics of thoracic rotation which demonstrates effectiveness for the amelioration of motor disabilities. When calculating the difference between sides, that is the asymmetry, the data of participants $3 \mathrm{~B}$ and $5 \mathrm{C}$ were excluded from the sample given a remaining asymmetry after treatment. For the calculation, the value of $V 3$ was subtracted from $V 4$. The asymmetry diminished from -9.08 to -1.09 degrees in Group 2, and from -5.89 to -0.44 degrees in Group 3, a mean reduction of -6.72 degrees $(t=$ $-7.05, p<0.01)$. This improvement in symmetry is evident in these two Groups, but less evident in Group 1. Using the same technique, the six elements remaining in Group 1 were insufficient for statistical analysis, probably due to non-homogeneity in their motor impairment (see Appendix, Note 3). Regarding another important effect, the amelioration of pain ( $V 7)$, in Group 1 the mean pain value at baseline improved significantly after treatment $(t=6.91, p<0.001)$, the mean values per sessions: 1) $3.92,2) 1.92,3) 1.46$, (Table 1). The difference between the first and second session is significant as well the difference between the first and the third (i-ii $t=6.67, p<0.001$; i-iii $t=8.21, p<0.001$ ). These results indicate that asymmetry in the thoracic rotation can act as a causal factor in motor disabilities and pain. However, results also demonstrate that the treatment is effective in resetting the default symmetry in the dynamics of thoracic rotation. 
Table 1. Indicate mean values. Variables $V 1, V 2, V 3, V 4$ indicate degrees of joint range of motion (ROM), $V 5$ and $V 6$ indicate the score of perceived exertion of effort, $V 7$ indicate the score of pain. Small letters, or the ${ }^{*}$, indicate the significant differences.

\begin{tabular}{|c|c|c|c|c|c|c|c|c|c|c|}
\hline \multicolumn{6}{|l|}{ Group 1} & \multicolumn{5}{|c|}{ Group 2 Group 3} \\
\hline before & after & & session I & session II & session III & & & before & after & \\
\hline \multicolumn{11}{|l|}{$V 1$} \\
\hline 123.33 & 127.72 & * & $124.81^{\mathrm{e}}$ & $126^{\mathrm{e}}$ & 125.77 & 122.49 & 118.89 & 119.96 & 121.42 & * \\
\hline \multicolumn{11}{|l|}{$V 2$} \\
\hline 57.54 & 59.79 & * & $55.27^{\mathrm{m}}$ & 58.77 & $61.96^{\mathrm{m}}$ & 59.85 & 57.38 & 57.92 & 59.31 & \\
\hline \multicolumn{11}{|l|}{$\sqrt{3}$} \\
\hline 73.21 & 73.77 & & 72.62 & 72.88 & 74.96 & 70.89 & 66.42 & 68.35 & 68.96 & \\
\hline \multicolumn{11}{|l|}{$V 4$} \\
\hline 74.92 & 78.23 & * & $75.08^{\mathrm{b}}$ & $75.85^{\mathrm{d}}$ & $78.81^{\text {bd }}$ & 67.52 & 65.83 & 64.42 & 68.92 & * \\
\hline \multicolumn{11}{|l|}{$\sqrt{5}$} \\
\hline 0.29 & -0.29 & * & $0.4^{\mathrm{g}}$ & $0.13^{\mathrm{h}}$ & $-0.53^{\text {gh }}$ & 0.14 & -0.14 & 0.21 & -0.21 & * \\
\hline \multicolumn{11}{|l|}{$V 6$} \\
\hline \multirow[t]{2}{*}{0.24} & -0.24 & * & $0.36^{1}$ & $-0.32^{1}$ & -0.04 & & $\begin{array}{c}V 6- \\
\text { Group } 2\end{array}$ & $0.66^{\mathrm{a}}$ & $-0.17^{\mathrm{a}}$ & \\
\hline & & & & & & & $\begin{array}{c}V 6- \\
\text { Group } 3\end{array}$ & -0.17 & -0.31 & \\
\hline$V 7$ & & & & & & & & & & \\
\hline 3.28 & 1.59 & * & $3.92^{\mathrm{jk}}$ & $1.92^{\mathrm{k}}$ & $1.46^{\mathrm{j}}$ & 1.83 & 0.94 & 2.04 & 0.73 & * \\
\hline
\end{tabular}

Variable $\boldsymbol{V}$, in Group 1, the difference in the maximum passive flexion on the neck is significant. Additionally, as described in Appendix (Note 3), all participants experienced an immediate amelioration of their motor impairments. For example, participants $4 \mathrm{~A}, 7 \mathrm{~A}$, and $12 \mathrm{~A}$ had been suffering a severe impairment to rotate the head toward the right. After the first section, their ability to rotate the head toward the right-side returned to the normal range of motion. Similar amelioration was also observed for other impairments. Thus, participants $1 \mathrm{~A}$ and 2A had been unable to flex the right-side shoulder. The observed after effect was an immediate return to normal ability to perform maximum flexion. Furthermore, in Group 1, the maximum degree of neck passive flexion reached saturation after the first session; i.e., the difference in $V 1$ is significant solely in the first session. The mean of 123.33 degrees at baseline rises after treatment to $127.72(t=-4.23, p<0.001)$. In Groups 2 and 3 , the mean of 119.96 degrees at baseline subsequently rises to $121.42(t=-2.35, p=$ 0.027). It is interesting that the ROM in Group 1 increases and that the baseline value of $V 1$ in Group 1 and the value after treatment in Groups 2 and 3 are similar.

In Group 1 , variable $\boldsymbol{V} \mathbf{2}$, the thoracic flexion incrementally increased after each session. The mean of 57.54 degrees at baseline, i.e., the thoracic passive flexion increases after treatment to $59.79(t=-2.04, p=0.046)$. Although the level of significance is close to $p 0.05$, with additional applications the difference 
is clearly observed. Consequently, the mean increment after sessions is: 1) 55.27 , 2) $58.77,3) 61.96$ degrees. Differences are significant between the first and second session $(t=-2.58, p=0.012)$, and the second and third $(t=-2.36, p=$ 0.022). On the other hand, Groups 2 and 3 failed to reach significance. These results shed some light on the problem of stability of the spine column and also demonstrate that passive flexion of the spine is important. Murray (1939) describe the mechanism for regulating the spinal foramen considering its dynamics for flexion and extension: "The articular process with the facets forms the posterior boundary of the intervertebral foramen for the passage of the anterior and posterior spinal nerves. When the spine in the lumbosacral region is forced into extension (that is, bending backwards) these facets override one another and diminish the vertical diameter of the foramen. When the lumbar spine is bent forward (flexion), the reverse takes place and the foramen is enlarged vertically, given more space for the spinal nerves". Here, the spinal foramen's geometry forms a mechanism that increases the stability of the spinal cord by narrowing the spinal foramen in dynamic modes such as walking or running. The spinal foramen naturally enlarges when the coordination mode does not challenge the centre of mass (COM) too strongly, as in a relaxed sitting position. Improvement in variable $\mathbf{V} \mathbf{2}$ was however the principal result of the improved efficiency of the OAM, achieved by the torsion of the spine rather than flexion or extension.

The value of variables $V 3$ and $V 4$ indicate the maximum active thoracic rotation when the pelvis is stationary. Interestingly variable $V 3$ did not attain significance for all three Groups; however, symmetrically variable $V \mathbf{4}$ did reach significance in all Groups. V4 is the left-side up thoracic rotation: in Group 1 the mean rotation of 74.92 degrees at baseline rises after treatment to 78.23 ( $t=$ $-2.63, p=0.011$ ). Thus, the mean rises in sessions: (i), (ii), (iii), respectively $75.08,75.85,78.81$ degrees. The difference between the first and third session is significant $(t=-2.42, p=0.019)$. In Groups 2 and 3 the mean of 64.42 degrees at baseline increases to $68.92(t=-3.11, p<0.01)$. These results suggest that the right-handed have a preference in thoracic rotation for task performance when the pelvis is stationary. The significant difference in $V 4$, but not in $V 3$, demonstrates the presence of considerable articular drift at baseline, this articular drift being an angle of joint rotation out of the controller radar. In only one side, insensitivity to tension of muscle spindles in the OAM is a malfunction which causes an articular drift (cf. Diederichs, 2017). During the preliminary interview, articular drift was assessed by passive rotation of the shoulder girdle from sitting. An active rotation, while the pelvis is stationary, as for variables $V 3$ and V4, would be a more reliable test. Additionally, Groups 2 and 3 performed the treatment with the dominant and non-dominant hand, respectively, and the after effect coincided fully, with the exception of how actors in Group 3 perceived effort when testing for the variable $\boldsymbol{V} \mathbf{6}$, see Figure 2.

The kinematic configuration for $\sqrt{5}$ and $V \mathbf{6}$ challenges the OAM isometrically 


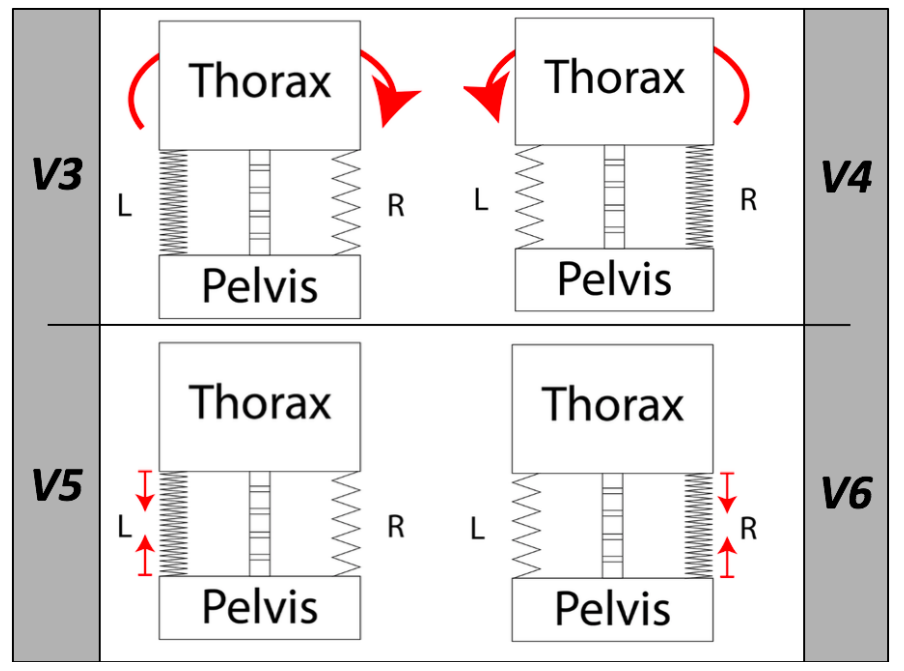

Figure 2. The diagram illustrates the oblique abdominal muscle (OAM) conditioning state according to the kinematic configuration for variables: $\mathbf{V} \mathbf{3}, \mathbf{V}, \boldsymbol{V} \mathbf{5}$, and $\mathbf{V 6}$. The dense zigzag lines indicate short-range stiffness of the OAM; the capital letters L and R indicate the left and right sides, respectively. The large arrows in $V 3$ and $V 4$ indicate the direction of movement. The two small arrows in $V 5$ and $\mathbf{V} \mathbf{6}$ indicate the short-range stiffness of the OAM, which breaks the acceleration of the centre of mass when assuming the kinematic configuration.

in one side only. The total generation of perceived effort might equal the ability of muscles to respond reflexively, i.e., a diminished perception of effort signifies more efficient reflexes for motor control. In Group 1, when testing the OAM in the left side, i.e. $V 5$, the perceived effort diminished from (0.29) before to $(-0.29)$ after $(t=3.79, p=0.001)$, in $\mathrm{z}$ scores. That indicates a difference in perceived effort from very hard to very easy. After each session the mean perception of effort drops: 1) 0.4 , 2) $0.13,3)-0.53$; differences are significant between the second and third session $(t=3.53, p<0.001)$, and between the first and third $(t$ $=4.97, p<0.001)$. In Groups 2 and 3, the difference before (0.21) and after $(-0.21)$ is significant $(t=2.63, p=0.014)$. Regarding V6: in Group 1, the perceived effort diminished from (0.24) before to $(-0.24)$ after $(t=2.84, p<0.001)$, in $\mathrm{z}$ scores. For every additional session the mean perceived effort falls: 1) 0.36 , 2) $-0.32,3)-0.04$; the difference is significant between the first and second session $(t=3.29, p<0.01)$. In Group 2, the difference is significant for before (0.66) and $(-0.17)$ after $(3.63, \mathrm{df}=24, p<0.001)$. On the contrary, Group 3 failed to reach significance where participants performed the treatment with the non-dominant hand, challenging the OAM on the right side. Between Groups 1 and 2 the level of perceived effort for sustaining the isometric position became significantly lower. In Group 3, failure to reach significance in variable $V 6$ indicates a dominance in one side OAM, the left side in the right-handed. Thus, a diminished perception of effort suggests that the kinematic configuration is more automatized (see also Monjo \& Forestier, 2018). The intent here is not to 
infer the origin of perceived effort (Monjo et al., 2018); but rather to understand OAM effectiveness when challenged.

\section{General Discussion}

The experimental treatment described employs a tensile force which generates unexpected disturbance, implying the ability to precisely regulate muscle forces to maintain the hand in position A. An increase in muscle forces, or joint impedance, is an effective strategy to maintain posture during unexpected disturbance, but excessive muscle force limits the ability to perform tasks that require force regulation ( $\mathrm{Hu}$ et al., 2017). In Hu et al.'s experiment, small displacement perturbations were used to quantify impedance during exertion of volitional elbow torques from $0 \%$ to $20 \%$ of maximum voluntary contraction (MVC) in two tasks. Actors were instructed either not to intervene (DNI) with the imposed perturbations or to explicitly intervene so as to minimize the influence of the perturbations on the elbow torque. In both tasks real-time visual feedback of elbow torque was available. They found that, at $10 \%$ or $20 \%$ of MVC, impedance was respectively $35( \pm 12) \%$ and $40( \pm 13) \%$ smaller when actors intervene; indicating that muscle force regulation was assisted also by a sensory feedback response; in the DNI task, impedance was increased which indicates only a feed-forward response (Hu et al., 2017). In the present study, actors reacted to the tensile force to maintain the hand in the maximum reachable amplitude, possible by generating prediction of the event to come in order to anticipate the response (Schmidt, 1968). However actors were not expecting their hand to accelerate as a result of the three DOFs of the shoulder, specifically of the glenohumeral joint (e.g., Haering et al., 2014). After the tensile force is transferred, the actor's hand accelerates (phase 3-Appendix, Note 5) and the RT for controlling the hand position (phase 4) ranges between a minimum of $\sim 150 \mathrm{~ms}$ and a maximum of $\sim 340 \mathrm{~ms}$, with a mean of $\sim 230 \mathrm{~ms}$. In addition, $\mathrm{Hu}$ et al. (2017) suggested that a sensory feedback response opposing the unexpected disturbance becomes available after $\sim 150 \mathrm{~ms}$, which is empirically consistent with the minimum RT found in the present study.

This indicates that the history dependent features of spindles in passive muscles maintained at a constant length during control of posture and balance are used to detect the sensorimotor response to perturbations (Blum et al., 2017). In a rapid shoulder flexion paradigm, while standing upright electromyography (EMG) was used to test activation patterns of both transverse abdominal (TrM) and internal oblique (IO) muscles; the authors found that EMG activation of the contralateral TrM-IO occurred prior to starting shoulder flexion (Massé-Alarie et al., 2012). That is, during a standing position the passive contralateral abdominal muscles are said to anticipate activation in order to counteract an imminent forceful shoulder flexion. In light of these finding, it would be reasonable to speculate that anticipation in the activation of contralateral abdominal muscles would limit the displacement of the COM. In the present study, to support 
maintaining the contralateral OAM passively at a constant length, the actor forcefully holds a stable handle while the pelvis is maintained stable by a belt. After a signal, the actor expects the transfer of the tensile force while retaining the knowledge of the importance to maintain position A. With transfer of the tensile force, the contralateral OAM reacts by maintaining its fibres' stiffness appropriately short, which causes a coupling between thorax and pelvis, i.e. a 1:1 phase-locked mode. Bear in mind that, initially, the lower limbs rest passively on the floor. The coupling from thorax to pelvis generates a qualitative reorganisation within segments of the lower limb resembling the midstance of gait (e.g., Kay \& Warren, 2001). This was often observed but not always, probably due to lack of normalisation of the tensile force.

Efficient short-range stiffness of the OAM is made possible as a result of muscle spindles acting as force-related variables (Blum et al., 2017). In the present study, actors undertook the treatment on one side only, the effect on the other side being observed as improved symmetry. Although only one hand was used, the effect of the treatment indicates that muscle spindles are recalibrated on both sides. Empirically, in Group 1 the stretch reflex was tested (at baseline - with participants standing upright) by pressing the OAM simultaneously on both sides. When comparing sides, an asymmetric reaction is observed, displaying larger latency in the right-side and, sometimes, the reaction is absent (see also Diederichs, 2017). Using EMG, Massé-Alarie et al. (2012) found a significantly larger latency in the contralateral abdominal muscles of a group suffering motor impairments, compared to the anticipated activation found in the control. In their experiment, participants performed rapid shoulder flexion while standing upright. In more natural coordinative modes, torsions assist the hand in reaching for and coupling with a target object and might promote the anticipated activation of muscles, too. There is good motivation for choosing the dominant hand for pairing the CS with US. In support is the overt facilitation that actors displayed in rotating the shoulder girdle towards one side (see Appendix, Note 1). In this example, the right-handed might exhibit a preference for performing torsions by using the left-side OAM to limit the displacement of the COM (e.g., Ellis et al., 2014). Thus, and especially in people suffering motor disability, this treatment is conveniently performed with the preferred hand, which challenges specifically the preferred OAM. The anticipation of contralateral OAM activation is also important for the stabilisation of the glenoid fossa of the scapula, which then allows the shoulder musculature to precisely regulate an effective force to control a hand's position coordinate when a disturbance occurs.

Actors maintain the spatial and temporal anticipation necessary, predicting stimulus arrival (Schmidt, 1968). Presentation of the US, that is the time it takes for a complete transfer of tensile force to the actor's hand, ranges from a minimum of $\sim 100$ ms to a maximum of $\sim 1.480 \mathrm{~ms}$, with a mean of $\sim 710 \mathrm{~ms}$. In this example, the maximum time is about $1.5 \mathrm{~s}$, which signifies that the actor took more time to anticipate sufficiently. The minimum is about $0.1 \mathrm{~s}$, signifying that 
anticipation was already sufficient to control the maximum reachable amplitude of the hand in the presence of the tensile force. Formally there is no RT when pairing CS and US. The difference between the moment that the tensile force is transferred and the time that the actor initiates the response is zero. Undeniably it would be disadvantageous to wait until the target event occurs, only then responding with a step impulse of maximum amplitude. Owing to inertia and delays in the nervous system, the actor must begin to respond before the tensile force is transferred. In that manner, prediction response rises to a level that coincides with the onset of the tensile force transfer (Moore \& Choi, 1997). In fact, actors are certain about the need to counteract a one-dimensional force vector in order to maintain position A, knowledge that was reinforced during the CS. Furthermore, actors received instruction to visually track their hands as visual feedback can help to issue a motor command (van Beers et al., 1999). After pairing CS and US, the amplitude which is the CR is maintained. The hand unexpectedly accelerates, drifting from the specified coordinate. Therefore, identification of the motor goal, i.e.: the specified coordinate in position A, is also possible by visual cue (Wong et al., 2015), see Figure 3. Thus after a reaction time (RT) a discrete movement is generated which returns the hand to the specified coordinate for a dwell time.

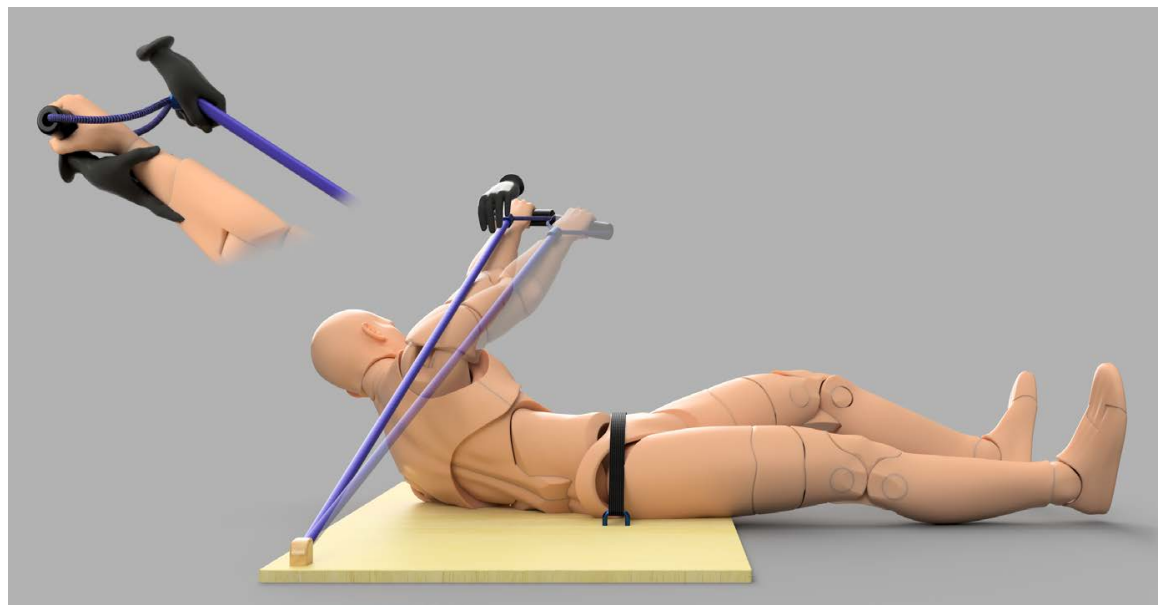

Figure 3. In the left upper corner, the moment before pairing CS with US is illustrated. The black hands are illustrating the clinician who holds the actor's wrist and the elastic tubing. The main illustration shows the moment after pairing CS with US; the more transparent limb illustrates the drifted position. Note that the actor still has a visual cue available, namely the clinician's hand at the specified coordinate, in position A. For pairing CS with US, first: the clinician releases the actor's wrist, just after giving a signal to start to counteract the tensile force (see Methodology), it is then transferred to the actor's hand. Thus, after hand stabilisation (see Appendix, Note 3.), the actor is instructed to release the arm to move the hand towards the shoulder while keeping the nose's position stationary. Stabilisation of the nose's position is possible by keeping a thumb touching the actor's nose, which ensures thoracic stability. Actors are instructed to track their hand visually. Often, in order to maintain the nose stationary, attention is attended by overt visual fixation while the actor's hand is attended as covert visual fixation (the latter signifies a target in the peripheral visual field), for a review see Castiello (2018). 
A CS event is an act of motor planning (Neige et al., 2018) which involves specification of the movement's trajectory, a description of how the end effector will produce a CS action, and the full set of joint trajectories or muscle activations required to execute the action (Wong et al., 2015). According to Moore and Choi (1997) "the onset of CS initiates a spreading pattern of activation among neurons tied to whatever sense modality is involved". They added that "a CS event initiates a cascade of activation such that one component excites that next, with some delay. When it does occur, a connection is established between elements of the cascade and the target. A representation of the target is evoked the next time these elements are activated" (Moore \& Choi, 1997). That is to say, a CS must be delivered in a way that counteracts the tensile force with a step impulse of maximum amplitude. The CS only instructs the actor to counteract a vertical force to maintain the hand in the specified coordinate position. After pairing CS with US, the clinician's hand is still visible to the actor and informs him or her about the centre of the coordinate that needs to be maintained (Figure 3). Therefore, an effective RT precisely regulates the muscle forces required to resolve one DOF of uncertainty as the learning process is optimised to pursue the goal, i.e. position A. Optimisation would shorten the confidence interval to process the hand's position and the centre of the coordinate that needs to be maintained (van den Berg et al., 2016).

An informative cue, i.e. a CS, represents a great degree of activation among the brain's cortical neurons as the primary source of motor planning (Neige et al., 2018); thus, pairing CS with US can facilitate a boost of intrinsic information toward the CNS as inputs of sensory transformation relative to the pelvic position (e.g., Faber et al., 2018). This question goes beyond the objectives of the present study and is therefore a matter for future research. During training, after a dwell time actors are instructed to release the arm and move the hand toward their shoulder: In Group 1, the observed movement is jerky, and the hand's trajectory more arched than that in control Group 2. An uncoordinated movement is observed between shoulder, elbow, and wrist. In spite of this, consider the training phase as a sequence of events in time divided into two major phases, i.e.: the initial phase, which starts with the paring of CS with US and ends in position A; then the final phase, which starts from position A and ends in position B. This is to say, this study restricts itself to the initial phase, i.e., from the pairing of CS with US until the end of the RT. Neige and others (2018), state that an RT is the outcome of the neuron process of five cortical regions integrated into a frontoparietal network for various aspects of motor planning. Thus the pairing of CS with US would reweigh the abstract representation of the body's segments among different clusters of cortical neurons (see also Tada et al., 2015). This would be optimised by the act of solving one DOF error by the issue of an effective discrete conditioned response ( $d \mathrm{CR}$ ), which is the RT's product, see Figure 3.

The CR entails a dynamic rearrangement throughout the body segments facilitating motor control to maintain a hand in the maximum reachable ampli- 
tude. Formally the $\mathrm{CR}$ is the motor rule, while for the motor goal the actor needs to discriminate between the specified coordinate and the drifted position. In order to attain the goal, i.e. position $\mathrm{A}$, the actor starts a sequence of actions simultaneously (Gallivan et al., 2016). In the following, the initial training phase is explained as the unfolding of events in time, its chronology is aligned with a self-organising neural network paradigm. First 1) the actor responds with a step impulse of maximum amplitude, which is the CR; in the next step 2) the actor deals with the consequence of an unexpected DOF; and then in the final step 3) the problem is solved through the issue of a $d \mathrm{CR}$. In a computational model, local discrimination of input is possible by recognising the neuron signature which reports both the neuron identity and the local informational context, i.e. a transient memory to keep track of the information and its source (Latorre et al., 2011). Latorre et al. define: a) the input to a neuron receptor consists of all messages at a given iteration; $b$ ) if the receptor detects in the message that data are relevant to solve the problem during processing, the receptor starts recognising the signature of the emitter neuron; c) if emitter and receptor data are compatible then the receptor neuron-which belongs to a cluster-starts recognising the corresponding signature of neurons in the cluster by reconfiguring the network (Latorre et al., 2011). Based on the treatment and results observed, the present author postulates that: i) iteration of neurons-considering the pairing of CS with US-is enhanced by the maximum size of the somatosensory information which is transmitted as input source; ii) the local informational context are memories of the body state (proprioceptive, haptic, and also exteroceptive), which report the relevant data to be processed; for then iii) resolve one DOF error by issuing an effective $d \mathrm{CR}$. The maximum size of somatosensory inputs might be the outcome of an efficient coupling between thorax and pelvis, which might be fundamental for motor learning (McNamee et al., 2016).

Using the maximum reachable amplitude, the actor learns a specific hand coordinate for sustaining a force relative to the pelvic position in which informational properties might be encoded in cortical neurons (Franklin et al., 2016; Stratmann et al., 2018). Fine, smooth coordination might be related to automation of relevant motor skills (e.g., Kal et al., 2018) in the cerebellum (Latorre et al., 2013; Martino et al., 2014). The treatment is characterised by incidental sequence learning, confirmed also by the fact that some actors reported verbally the sequential regularities of events, i.e., the drifted position. Verbal report is stated as the most sensitive test for conscious sequence knowledge defining sequence learning in a serial reaction time (SRT) task (Rünger \& Frensch, 2010). In Group 1, participants were randomly assigned independently of the location of their motor impairment, which suggests the treatment's generalisability. The asymmetric thoracic rotation and jerky upper limb movement observed in Group 1 are similar to the quality of movement observed in a seventy years old (right-handed) person two years after a left cerebral hemisphere stroke (raw data to appear in an upcoming publication). Clinically, it should be noted that, despite this observation only taking into account a single case, the experimental 
treatment-which here is termed an Elemental Conditioning Stimulus (ECS) approach-demonstrated broad efficacy in improving equilibrium and gait in a hemiplegic patient previously unable to walk without the aid of a rollator. The position required for application of the treatment seems ideal for patients suffering neurological symptoms. This paper however provides remarkable evidence for an effective treatment of motor disabilities. To date, it appears that no previous treatment based on classical conditioning has claimed to demonstrate rehabilitating motor disabilities efficiently (for a review see Raffin \& Hummel, 2018). An independent study is required to verify the efficacy of the ECS approach both in the neurologically intact population as well as in the hemiplegic population.

\section{Conclusion}

Empirically, the ECS approach is able to trigger a mechanism that detects a critical value and initiates a transition from the dynamics of the action system and task constraints to a default value (Diedrich \& Warren, 1998). This is clearly observed in the improvement of symmetry in Groups 2 and 3 and in the amelioration of motor disabilities and pain in Group 1. During training, the actor learns through the explicit memory system how to control a one-dimensional force vector by maintaining the hand in the maximum reachable amplitude, in position A. In the following moment, due to a sudden change in coordinate of the position of the hand, the actor incidentally learns how to precisely regulate the muscle forces to control only one degree of freedom through the implicit memory system (e.g., Steenbergen et al., 2010). The learning process in the implicit memory system precedes explicit learning, generating memory representations that directly control behavior (Rünger \& Frensch, 2010). Most significantly, implicit memory improves task performance by reducing both the average RT and the error rate (Rünger \& Frensch, 2010; van den Berg et al., 2016). With the first treatment application, the after effect-clearly observed in variables $\boldsymbol{V} \mathbf{1}$ and $\boldsymbol{V} \mathbf{2}$-is amelioration in muscle tone (see also Ivanenko \& Gurfinkel, 2018). Further amelioration is generally observed a day to even three weeks after treatment (Laventure et al., 2018). The final change in muscle tone is referred to as a positive feeling and often described by patients as "openness". In principle, implicit memory can store information regarding a learned size scaling (Hyun Geun et al., 2018) which might be used to generate motor schemes (Miranda et al., 2018). Scaling of muscle synergy appears to be elemental in motor control (d'Avella \& Lacquaniti, 2013; Israely et al., 2018; Singh et al., 2018).

The seven variables of the results also indicate generalisability. That is, the treatment can also be applied to ameliorate the symptomatology of motor disabilities related to an impairment in the nervous system employed to fine control movement and position, such as the proprioceptive system. Following this, so-called idiopathic diseases related to an impaired movement can also originate as the consequence of an impairment which first strikes the motor control systems rather than structural components of the musculoskeletal system. Conven- 
tional treatments target the structural tissues to tackle symptoms associated with orthopedic conditions, for example herniation of the intervertebral disc, the thoracic outlet syndrome or pain and impediment in other compartments of the body. However, mounting evidence suggests that a treatment based on the learning process can be considered the approach to adopt for amelioration of impairments related to motor control. Above I postulate that treatments to improve the functioning of the motor control systems can only be based on the learning process. On a clinical note, the ECS approach also demonstrates effectiveness in successfully rehabilitating a 43-year-old patient following a decompressive laminectomy of the cervical vertebrae ( $\mathrm{C} 2$ to $\mathrm{C} 7$ ) as a result of a previous cerebellar stroke. Effectiveness of the treatment however depends significantly on how the patient interprets the action she or he needs to perform, and for this it is important that the clinician be trained in apply learning process. The total tensile force for the treatment should be sufficient to stimulate a kinematic reaction of all segments of the body. This can be easily achieved gradually. For practise and pre-treatment purposes, the ECS approach also shows efficacy even when a lighter tensile force is used.

\section{Acknowledgements}

My thanks to Rafael Bernardes Ribas Gentile for establishing the precise chronology of the treatment-that is, the elapsed time between CS and US-from video records using Kinovea software. My thanks also to Marco Bressan for data input and for assistance with organization of the statistical results and to Andrea Merlo for our conversations and discussions on scientific methodology. Saif Mahmoud Mohamed provided invaluable technical support in generating the rendering, Figure 1 and Figure 3, and also proofreading the figure captions. Margaret Kneller and Lewis Samuel Klausner have my gratitude for commenting and proofreading the paper. And, of course, the author is grateful to James Ruscoe for his comments and then proofreading the final paper.

This work, however, would not have been possible without active participants and here I want personally to thank each one of you. Thanks go to Gabriele Simoncini for acute feedback. Additional well-merited thanks go to my patients whose motor abilities are so remarkably well adapted after a polio virus infection (poliomyelitis) in their early lives during the 1960s. Without these empirical observations commencing in 2007, the present work would never have seen light of day.

\section{Conflicts of Interest}

The author declares no conflicts of interest regarding the publication of this paper.

\section{References}

Amazeen, P. G., Amazeen, E. L., \& Turvey, M. T. (1998). Dynamics of Human Interseg- 
mental Coordination: Theory and Research. In D. A. Rosenbaum, \& C. E. Collyer (Eds.), Timing of Behavior: Neural, Psychological, and Computational Perspectives (pp. 237-259). Cambridge, MA: The MIT Press.

Aune, T. K., Aune, M. A., Ingvaldsen, R. P., \& Vereijken, B. (2017). Transfer of Motor Learning Is More Pronounced in Proximal Compared to Distal Effectors in Upper Extremities. Frontiers in Psychology, 8, 1530. https://doi.org/10.3389/fpsyg.2017.01530

Bates, D., Mächler, M., Bolker, B. et al. (2015). Fitting Linear Mixed-Effects Models Using lme4. Journal of Statistical Software, 67, 1-48. https://doi.org/10.18637/jss.v067.i01

Bernshtein, N. A. (1967). The Co-Ordination and Regulation of Movements. Oxford: Pergamon Press.

Blum, K. P., Lamotte D’Incamps, B., Zytnicki, D. et al. (2017). Force Encoding in Muscle Spindles during Stretch of Passive Muscle. PLOS Computational Biology, 13, e1005767. https://doi.org/10.1371/journal.pcbi.1005767

Borg, G. (1998). Ch. 7. Borg's Perceived Exertion and Pain Scales. In Administration of the Borg Scales (pp. 49-52). Champaign, IL: Human Kinetics.

Borg, G. A. (1982). Psychophysical Bases of Perceived Exertion. Medicine \& Science in Sports \& Exercise, 14, 377-381. https://doi.org/10.1249/00005768-198205000-00012

Caillou, N., Nourrit, D., Deschamps, T., Lauriot, B., \& Delignieres, D. (2002). Overcoming Spontaneous Patterns of Coordination during the Acquisition of a Complex Balancing Task. Canadian Journal of Experimental Psychology/Revue canadienne de psychologieexpérimentale, 56, 283-293. https://doi.org/10.1037/h0087404

Castiello, U. (2018). Ch. 9: Reach-to-Grasp Movements: Testing the Cognitive Architecture of Action. In Reach-to-Grasp Behavior: Brain, Behavior, and Modelling across the Life Span (pp. 197-223). Boca Raton, FL: CRC Press. https://doi.org/10.4324/9780429467875-9

d'Avella, A., \& Lacquaniti, F. (2013). Control of Reaching Movements by Muscle Synergy Combinations. Frontiers in Computational Neuroscience, 7, 42. https://doi.org/10.3389/fncom.2013.00042

Diederichs, V. M. (2017). A New Approach That Improves Range of Motion without Stretching or Pain Comparison with PNF. Functional Neurology, Rehabilitation, and Ergonomics, 7, 48-53.

Diederichs, V. M. (2018). A Novel Approach That Ameliorates Motor Disabilities and Improves Range of Motion of Joints; Comparison with Proprioceptive Neuromuscular Facilitation. American Journal of Sports Science and Medicine, 6, 15-21.

https://doi.org/10.12691/ajssm-6-1-4

Diedrich, F. J., \& Warren, W. H. (1998). Ch. 14: Dynamics of Human Gait Transitions. In D. A. Rosenbaum, \& C. Colley (Eds.), Timing of Behavior: Neural, Psychological, and Computational Perspectives (pp. 323-343). Cambridge, MA: The MIT Press.

Ellis, R. G., Sumner, B. J., \& Kram, R. (2014). Muscle Contributions to Propulsion and Braking during Walking and Running: Insight from External Force Perturbations. Gait Posture, 40, 594-599. https://doi.org/10.1016/j.gaitpost.2014.07.002

Faber, H., Soest, A. J., \& Kistemaker, D. A. (2018). Inverse Dynamics of Mechanical Multibody Systems: An Improved Algorithm That Ensures Consistency between Kinematics and External Forces. PLoS ONE, 13, e0204575.

https://doi.org/10.1371/journal.pone.0204575

Fitts, P. M. (1954). The Information Capacity of the Human Motor System in Controlling the Amplitude of Movement. Journal of Experimental Psychology, 47, 381-391. https://doi.org/10.1037/h0055392 
Franklin, D. W., Batchelor, A. V., \& Wolpert, D. M. (2016). The Sensorimotor System Can Sculpt Behaviorally Relevant Representations for Motor Learning. eNeuro, 3, e0070-16. https://doi.org/10.1523/ENEURO.0070-16.2016

Gallivan, J. P., Bowman, N. A., Chapman, C. S., Wolpert, D. M., \& Flanagan, J. R. (2016). The Sequential Encoding of Competing Action Goals Involves Dynamic Restructuring of Motor Plans in Working Memory. Journal of Neurophysiology, 115, 3113-3122. https://doi.org/10.1152/jn.00951.2015

Gritsenko, V., Hardesty, R. L., Boots, M. T., \& Yakovenko, S. (2016). Biomechanical Constraints Underlying Motor Primitives Derived from the Musculoskeletal Anatomy of the Human Arm. PLoS ONE, 11, e0164050.

https://doi.org/10.1371/journal.pone.0164050

Haering, D., Raison, M., \& Begon, M. (2014). Measurement and Description of Three-Dimensional Shoulder Range of Motion with Degrees of Freedom Interactions. Journal of Biomechanical Engineering, 136, Article ID: 084502. https://doi.org/10.1115/1.4027665

Holt, K. G., Wagenaar, R. O., \& Saltzman, E. (2010). A Dynamic Systems: Constraints Approach to Rehabilitation. Brazilian Journal of Physical Therapy, 14, 446-463. https://doi.org/10.1590/S1413-35552010000600002

Hu, X., Ludvig, D., Murray, W. M. et al. (2017). Using Feedback Control to Reduce Limb Impedance during Forceful Contractions. Scientific Reports, 7, Article No. 9317. https://doi.org/10.1590/S1413-35552010000600002

Hyun Geun, S., Youn-Seok, L., \& Sang Jeong, K. (2018). The Emerging Concept of Intrinsic Plasticity: Activity-Dependent Modulation of Intrinsic Excitability in Cerebellar Purkinje Cells and Motor Learning. Experimental Neurobiology, 27, 139-154. https://doi.org/10.5607/en.2018.27.3.139

Israely, S., Leisman, G., \& Carmeli, E. (2018). Neuromuscular Synergies in Motor Control in Normal and Poststroke Individuals. Reviews in the Neurosciences, 29, 593-612. https://doi.org/10.1515/revneuro-2017-0058

Ivanenko, Y., \& Gurfinkel, V. S. (2018). Human Postural Control. Frontiers in Neuroscience, 12, 171. https://doi.org/10.3389/fnins.2018.00171

Kal, E., Prosée, R., Winters, M., \& van der Kamp, J. (2018). Does Implicit Motor Learning Lead to Greater Automatization of Motor Skills Compared to Explicit Motor Learning? A Systematic Review. PLoS ONE, 13, e0203591. https://doi.org/10.1371/journal.pone.0203591

Kay, B. A., \& Warren, W. H. Jr. (2001). Coupling of Posture and Gait: Mode Locking and Parametric Excitation. Biological Cybernetics, 85, 89-106. https://doi.org/10.1007/PL00008002

Kourtidou-Papadeli, C., Papadelis, C. L., Vernikos, J., Bamidis, P. D., Hitoglou-Antoniadou, M., Perantoni, E., \& Vlachogiannis, E. (2008). The Therapeutic Benefits of Gravity in Space and on Earth. Hippokratia, 12, 28-31.

Latorre, R., Aguirre, C., Rabinovich, M. I. et al. (2013). Transient Dynamics and Rhythm Coordination of Inferior Olive Spatio-Temporal Patterns. Front Neural Circuits, 7, 138 https://doi.org/10.3389/fncir.2013.00138

Latorre, R., de Borja, R. F., \& Varona, P. (2011). Signature Neural Networks: Definition and Application to Multidimensional Sorting Problems. IEEE Transactions on Neural Networks, 22, 8-23. https://doi.org/10.1109/TNN.2010.2060495

Laventure, S., Pinsard, B., Lungu, O. et al. (2018). Beyond Spindles: Interactions between Sleep Spindles and Boundary Frequencies during Cued Reactivation of Motor Memory 
Representations. Sleep, 41, zsy142. https://doi.org/10.1093/sleep/zsy142

Martino, G., Ivanenko, Y. P., Serrao, M. et al. (2014). Locomotor Patterns in Cerebellar Ataxia. Journal of Neurophysiology, 112, 2810-2821. https://doi.org/10.1152/jn.00275.2014

Massé-Alarie, H., Flamand, V.H., Moffet, H. et al. (2012). Corticomotor Control of Deep Abdominal Muscles in Chronic Low Back Pain and Anticipatory Postural Adjustments. Experimental Brain Research, 218, 99. https://doi.org/10.1007/s00221-012-3008-9

McNamee, D., Wolpert, D. M., \& Lengyel, M. (2016). Efficient State-Space Modularization for Planning: Theory, Behavioral and Neural Signatures. In 30th Conference on Neural Information Processing Systems (pp. 4511-4519). Barcelona: NIPS.

Miranda, J. G. V., Daneault, J.-F., Vergara-Diaz, G. et al. (2018). Complex Upper-Limb Movements Are Generated by Combining Motor Primitives That Scale with the Movement Size. Scientific Reports, 8, Article No. 12918.

https://doi.org/10.1038/s41598-018-29470-y

Monjo, F., \& Forestier, N. (2018). Muscle Spindle Thixotropy Affects Force Perception through Afferent Induced Facilitation of the Motor Pathways as Revealed by the Kohnstamm Effect. Experimental Brain Research, 236, 1193-1204.

https://doi.org/10.1007/s00221-018-5207-5

Monjo, F., Shemmell, J., \& Forestier, N. (2018). The Sensory Origin of the Sense of Effort Is Context-Dependent. Experimental Brain Research, 236, 1997. https://doi.org/10.1007/s00221-018-5280-9

Moore, J. W., \& Choi, J. S. (1997). Conditioned Response Timing and Integration in the Cerebellum. Learning \& Memory, 4, 116-129. https://doi.org/10.1101/1m.4.1.116

Murray, J. M. (1939). Lower Back Pain. Canadian Medical Association Journal, 41, 427-434.

Neige, C., Massé-Alarie, H., \& Mercier, C. (2018). Stimulating the Healthy Brain to Investigate Neural Correlates of Motor Preparation: A Systematic Review. Neural Plasticity, 2018, Article ID: 5846096. https://doi.org/10.1155/2018/5846096

Newell, K. M., \& van Emmerik, R. E. (1989). The Acquisition of Coordination: Preliminary Analysis of Learning to Write. Human Movement Science, 8, 17-32. https://doi.org/10.1016/0167-9457(89)90021-3

Pageaux, B. (2016). Perception of Effort in Exercise Science: Definition, Measurement and Perspectives. European Journal of Sport Science, 16, 885-894. https://doi.org/10.1080/17461391.2016.1188992

Puig-Diví, A., Padullés-Riu, J. M., Busquets-Faciaben, A. et al. (2017). Validity and Reliability of the Kinovea Program in Obtaining Angular and Distance Dimensions. Preprints, 2017, Article ID: 2017100042. https://doi.org/10.20944/preprints201710.0042.v1

Raffin, E., \& Hummel, F. C. (2018). Restoring Motor Functions after Stroke: Multiple Approaches and Opportunities. The Neuroscientist, 24, 400-416. https://doi.org/10.1177/1073858417737486

Rünger, D., \& Frensch, P. A. (2008). How Incidental Sequence Learning Creates Reportable Knowledge: The Role of Unexpected Events. Journal of Experimental Psychology: Learning, Memory, and Cognition, 34, 1011-1026. https://doi.org/10.1037/a0012942

Rünger, D., \& Frensch, P. A. (2010). Defining Consciousness in the Context of Incidental Sequence Learning: Theoretical Considerations and Empirical Implications. Psychological Research, 74, 121-137. https://doi.org/10.1007/s00426-008-0225-8

Schmidt, R. A. (1968). Anticipation and Timing in Human Motor Performance. Psychological Bulletin, 70, 631-646. https://doi.org/10.1037/h0026740 
Schmidt, R. A., Zelaznik, H., Hawkins, B. et al. (1979). Motor-Output Variability: A Theory for the Accuracy of Rapid Motor Acts. Psychological Review, 86, 415-451. https://doi.org/10.1037/0033-295X.86.5.415

Seay, J. F., Emmerik, R. E., \& Hamill, J. (2011). Influence of Low Back Pain Status on Pelvis-Trunk Coordination during Walking and Running. Spine, 36, E1070-9. https://doi.org/10.1097/BRS.0b013e3182015f7c

Singh, R. E., Iqbal, K., White, G. et al. (2018). A Systematic Review on Muscle Synergies: from Building Blocks of Motor Behavior to a Neurorehabilitation Tool. Applied Bionics and Biomechanics, 2018, Article ID: 3615368. https://doi.org/10.1155/2018/3615368

Steenbergen, B., van der Kamp, J., Verneau, M., Jongbloed-Pereboom, M., \& Masters, R. S. (2010). Implicit and Explicit Learning: Applications from Basic Research to Sports for Individuals with Impaired Movement Dynamics. Disability and Rehabilitation, 32, 1509-1516. https://doi.org/10.3109/09638288.2010.497035

Stratmann, P., Albu-Schäffer, A., \& Jörntell, H. (2018). Scaling Our World View: How Monoamines Can Put Context into Brain Circuitry. Frontiers in Cellular Neuroscience, 12, 506. https://doi.org/10.3389/fncel.2018.00506

Tada, M., Nishizawa, M., \& Onodera, O. (2015). Redefining Cerebellar Ataxia in Degenerative Ataxias: Lessons from Recent Research on Cerebellar Systems. Journal of Neurology, Neurosurgery \& Psychiatry, 86, 922-928. https://doi.org/10.1136/jnnp-2013-307225

Tanigawa, A., Morino, S., Aoyama, T. et al. (2018). Gait Analysis of Pregnant Patients with Lumbopelvic Pain Using Inertial Sensor. Gait \& Posture, 65, 176-181. https://doi.org/10.1016/j.gaitpost.2018.07.165

van Beers, R. J., Sittig, A. C., \& Gon, J. J. (1999). Integration Proprioceptive and Visual Position-Information: An Experimentally Supported Model. Journal of Neurophysiology, 81, 1355-1364. https://doi.org/10.1152/jn.1999.81.3.1355

van den Berg, R., Zylberberg, A., Kiani, R., Shadlen, M. N., \& Wolpert, D. M. (2016). Confidence Is the Bridge between Multi-Stage Decisions. Current Biology, 26, 3157-3168. https://doi.org/10.1016/j.cub.2016.10.021

van den Hoorn, W., Bruijn, S. M., Meijer, O. G. et al. (2012). Mechanical Coupling between Transverse Plane Pelvis and Thorax Rotations during Gait Is Higher in People with Low Back Pain. Journal of Biomechanics, 45, 342-347.

https://doi.org/10.1016/j.jbiomech.2011.10.024

Van Dillen, L. R., Sahrmann, S. A., Caldwell, C. A. et al. (2006). Trunk Rotation-Related Impairments in People with Low Back Pain Who Participated in 2 Different Types of Leisure Activities: A Secondary Analysis. Journal of Orthopaedic \& Sports Physical Therapy, 36, 58-71. https://doi.org/10.2519/jospt.2006.36.2.58

van Drunen, P., van der Helm, F. C. T., van Dieën, J. H. et al. (2016). Trunk Stabilization during Sagittal Pelvic Tilt: From Trunk-on-Pelvis to Trunk-in-Space Due to Vestibular and Visual Feedback. Journal of Neurophysiology, 115, 1381-1388.

https://doi.org/10.1152/jn.00867.2015

Wong, A. L., Haith, A. M., \& Krakauer, J. W. (2015). Motor Planning. The Neuroscientist, 21, 385-398. https://doi.org/10.1177/1073858414541484 


\section{Appendix}

1) A preliminary assessment for asymmetry was performed as part of the inclusion criteria. Assessed when sitting, participants were asked to keep their hands touching the ipsilateral shoulder while the clinician gently rotates the participant shoulder-girdle to the sides. The test verifies the maximum passive rotation of the shoulder-girdle while the pelvis is stationary. To verify the validity of this preliminary assessment, the baseline value of variables V3 and V4 was used. In comparison the asymmetry was coherent in twenty out of twenty-six cases in Groups 2 and 3, while in Group 1 this asymmetry was coherent only in five cases.

2) To select right-handed participants, the following questions were asked during the preliminary interview. The exclusion criteria were (i) no preference between sides to perform fine movement as indicated in questions (i) and (ii) or to have been trained to use the right-side hand during the infancy, question (iii). Thus, to be included in this study, participants must use only the right hand to brush their teeth and write.

a) "Do you prefer to use the right or the left hand to brush your teeth?"

b) "Do you prefer to use the right or the left hand to write?"

c) "Have you been taught to use the right hand because when young you were left-handed?"

3) The baseline condition of participants was described as follows. In Group 1, once the painful joint angle is indicated, the joint angle is photographed, and pain scored on a numeric scale. After treatment, pain was scored again with the joint at exactly the same angle as before treatment for comparison. Specifically, regarding the shoulder, participants $1 \mathrm{~A}$ and $2 \mathrm{~A}$ were suffering severe impairment, and $11 \mathrm{~A}$ mild impairment in flexing the right shoulder, while $10 \mathrm{~A}$ displayed mild impairment to flex the left shoulder. Participants described here as suffering severe impairment signifies that they could not raise their hand over the shoulder; while mild impairment means some limitation in shoulder flexion. In order to score pain, they indicated the highest position of their hand-while keeping the elbow locked-and then the sensation of pain was scored. For the neck, participants were asked to indicate the maximum rotation on both sides. Participants $4 \mathrm{~A}, 7 \mathrm{~A}$ and $12 \mathrm{~A}$ were suffering a severe impairment, while $5 \mathrm{~A}, 8 \mathrm{~A}$ and $13 \mathrm{~A}$ were suffering a mild impairment to rotate towards the right side. $\mathrm{A}$ severe impairment in this case signifies that rotation to that side is completely impeded, while mild impairment indicates some limitation to rotate the head. Participant $6 \mathrm{~A}$ was suffering severe impairment of the wrist in extending the hand over the forearm. This very extension was used to score pain. Participant $3 \mathrm{~A}$ was suffering moderate impairment in the thorax which she described as "feeling locked" when attempting to rotate towards the right side. This very rotation was used to score pain before and after treatment. Participant 9A was suffering mild impairment to flex the lumbar spine. This very flexion of the lumbar spine was used to score pain, using the same criteria as above, before and after 
treatment.

In Groups 2 and 3, participants were healthy and able to perform motor tasks normally. Pain was scored for specific movements, comparing the right and left sides of the body. To compare and score how comfortable it is to use the right and left sides of the body, participants attempted symmetrical movements, in both sides, to test the feeling. For example, 1) the squat test was performed by keeping the shoulders in maximum flexion, elbows locked, then bending the knees while keeping the heels on the floor; 2) the maximum extension of the lumbopelvic compartment was tested by pushing the pelvis forward while keeping knees locked and hands on the pelvic girdle; 3) with knees locked, shifting the pelvis to both sides was performed from the maximum extension of the lumbopelvic compartment, that is, by pushing the pelvis maximally forward and then also toward the sides; 4) while keeping knees locked, the thorax was rotated maximally toward both sides; 5) maximum flexion of the shoulder, while keeping knees locked and pelvis in maximum extension, was tested on both sides for comparison. These tests should be performed from standing upright. And while testing the movement, low speed and the maximum available amplitude are preferred because this provides the opportunity to perceive differences between the sides. That is, differences in perceived comfort or pain when approaching certain amplitude can easily be found and scored by comparison with the contralateral compartment. By testing the quality of a slow movement at maximum amplitude in both sides, painful angles are easily scored. Once a compartment is indicated as a source of pain, the joint angle is photographed, and the same joint angle is verified after treatment. For each participant, the painful compartment was the following: for participants $1 \mathrm{~B}, 11 \mathrm{~B}, 2 \mathrm{C}, 8 \mathrm{C}$, the right-side shoulder; for $2 \mathrm{~B}$ and $5 \mathrm{~B}$, the left-side shoulder; for $6 \mathrm{~B}, 7 \mathrm{~B}, 6 \mathrm{C}, 8 \mathrm{C}, 9 \mathrm{C}, 10 \mathrm{C}$ and $12 \mathrm{~B}$, the neck; for $11 \mathrm{C}$, $12 \mathrm{C}, 13 \mathrm{C}, 4 \mathrm{C}, 5 \mathrm{C}, 4 \mathrm{~B}$ and $9 \mathrm{~B}$, the lumbar spine; for $8 \mathrm{~B}$ and $10 \mathrm{~B}$, the right-side knee; and for $3 \mathrm{C}$, the thorax. Participants $1 \mathrm{C}, 3 \mathrm{~B}$ and $13 \mathrm{~B}$ had no complaints.

4) In standardising the procedure for data collection, and for participants better to understand "what to do", the instructions below were used:

$V 1$, from a sitting position, the participant is asked to imagine that his/her hands are tied up tightly to their knees. That is, with hands in a prone position above the knees holding and stabilising the upper thorax, while flexing the lumbosacral region maximally. Initially, in order to understand how to perform the maximum flexion of the lumbosacral region: the participant is asked to imagine that the clinician's hand is a "cactus' plant with spines" which is approaching his/her stomach while their hands are tied to their knees. In a first attempt the clinician uses one hand to gently push the participant's abdominal compartment backwards, the other is placed above vertebra T1 guiding and ensuring lumbosacral region maximum flexion. Once understanding what is needed, the participant is requested again to perform a maximum lumbosacral flexion. In this manner the participant learns how to perform the maximum flexion of the lumbosacral region while maintaining the hands stationary on top of the knees while sitting. For collecting data, rather than forcing the spine backwards, the 
final position is assumed with the musculature completely relaxed which ensures the lumbosacral region maximum and natural flexion. The participant is then instructed to keep his/her elbows bent resting on top of the thighs, hands in a supine position. This position ensures a natural passive flexion of the spine, possible by using the upper limbs as stabilisers ensuring stabilisation of the upper thorax. Data is collected from the first thoracic vertebra and top of the skull.

$V \mathbf{2}$, from standing upright, the participant is asked to imagine two arrows pointing in opposite directions: one arrow pointing toward the posterior compartment of the knees; the other pointing toward the anterior compartment of the pelvis. These imaginary arrows must be of equal force, thus ensuring stabilization of the pelvis by a fine contraction of the musculature. First, the clinician must ascertain that the participant's knees are locked in maximum extension for then guiding the participant's neck in maximum flexion. Second, for guiding the participant's neck in maximum flexion, it is important to keep the clinician's free hand in the participant's lumbopelvic region to ensure that it is not moving backwards. For the final position, the knees and the lumbosacral region must be stable in extension; the clinician then guides the participant's neck in maximum passive flexion by placing a hand on the top of the participant's head, asking $\mathrm{him} /$ her to release the head downwards, i.e. to move the chin towards the chest (instruction is given to maintain their jaw comfortably closed). For collecting data, the participant stands upright, with one side toward a wall bar used to support one forearm in a supine position. This helps the participant to keep his/her position more stable. Thus, the degree of inclination of the first and the last thoracic vertebrae is collected.

Variables $V 3$ and $V 4$. From a quasi-crawling position, the participant's elbow which is supporting the upper body must closely touch the ipsilateral knee, while the other hand is on the nape of the neck (see Figure 4). By using one hand, the clinician firmly presses the participant's pelvis downwards ensuring the stability of the pelvis. In order not to exacerbate the natural torsional barrier, instruction is given to move slowly. The participant's objectives are to point the elbow toward the ceiling (while the other elbow is stationary) and to stop and hold the position at the movement's barrier. The first objective helps to maintain the shoulder girdle more stable for the performance of the thoracic rotation; the second helps to collect more reliable data. N.B. ballistic and jerky movements are considered invalid. Thus, if the participant performs a fast movement during the procedure then (s)he receives instruction to repeat in a slower manner in the next attempt. Data is collected only when the participant reaches the final position smoothly. The movement barrier is the final position which is held for a moment. Therefore, in order to collect data, it is essential that the final position is achieved by a controlled slow movement of maximum amplitude. A video illustrating the variables and also the treatment can be found in the online version of this paper.

Instruction given to score perceived effort. In Group 1, participants received the following instruction for scoring the variables $\sqrt{5}$ and $\sqrt{6}$. They were instructed 


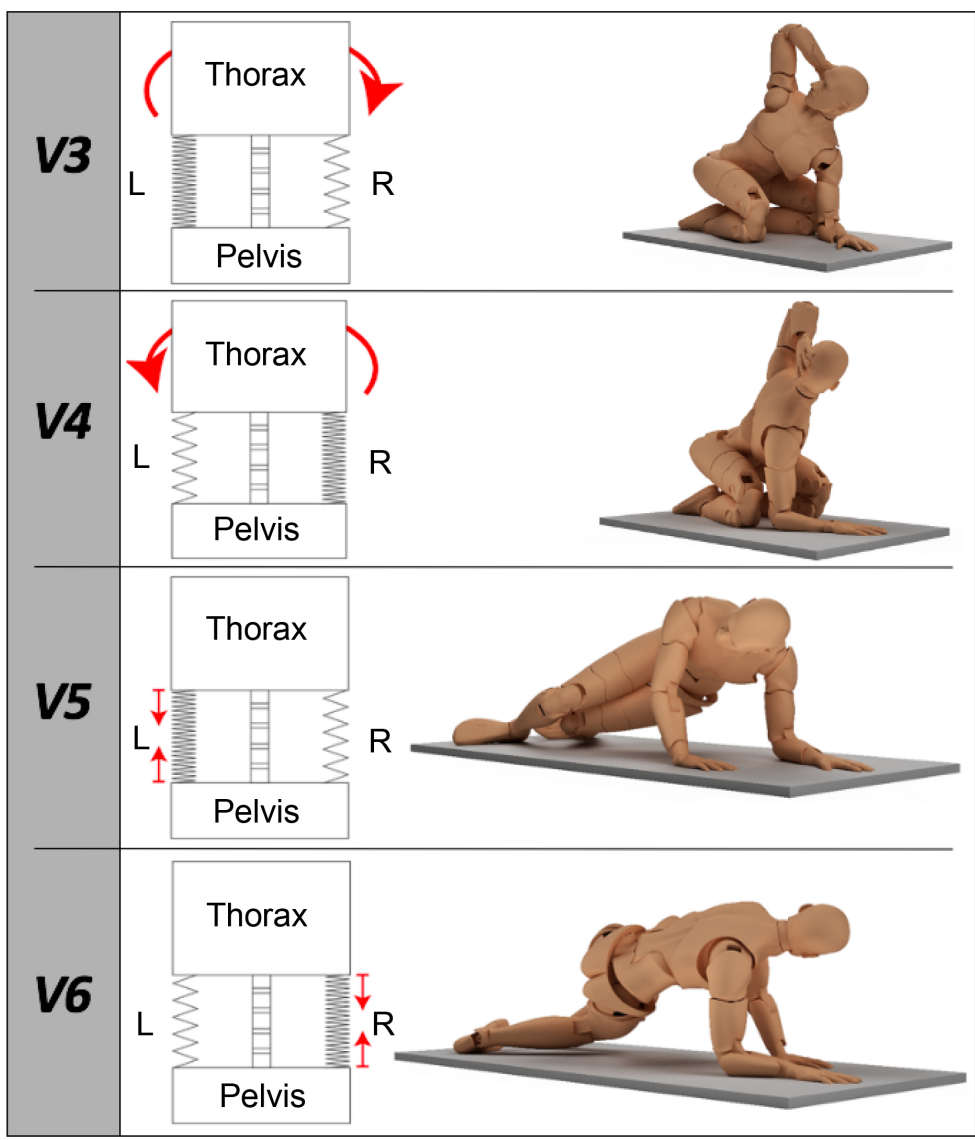

Figure 4. Diagrams (left) and the respective kinematic configuration (right). In the diagrams, the dense zigzag lines indicate short-range stiffness of the OAM, and the capital letters $\mathrm{L}$ and $\mathrm{R}$ indicate left and right sides, respectively. To better understand the conditioning state of the OAM, the diagram on the left and the actor's kinematic configuration should be compared. The large arrows in $V 3$ and $V 4$ indicate the direction of movement. The two small arrows in $\sqrt{5}$ and $\sqrt{6}$ indicate the short-range stiffness of the OAM which breaks the acceleration of the COM when assuming the kinematic configuration.

to score their sensation of effort, "how hard is to hold an isometric position", for the period during the exercise. That is, just the feeling experienced during the exercise (please see the work of Pageaux (2016) on how to instruct participants to score perceived effort). For the purpose of this study, participants were instructed that: "this is a very important test and focussing attention during the procedure is essential because it is very difficult to score effort sensation. Please, make sure that you clearly understand the position to be assumed and how to score effort. This signifies that if there is any doubt you should communicate that now because while performing the test, we need to maintain silence". The only communication during the procedure was to remind participants to "keep breathing". The instruction given to participants to score how hard is to hold an isometric position was "the amount of mental or physical energy required to hold the position." The ratings to score effort were explained by visual and ver- 
bal instruction: 1 , resting; 2 , very easy; 3 , easy; 4 , moderate; 5 , challenging; 6 , hard; 7 , slightly harder; 8 , very hard; 9 , really very hard; and 10 , maximum effort. Participants were explained that: "the mental and physical effort made to perform a task might be measurable by repeating precisely the same position in one side and then in the other side of the body". Variables $V \mathbf{5}$ and $\mathbf{V} \mathbf{6}$ are designed to allow isometrically identical positions in both sides.

Before testing the perceived effort, participants had the chance to familiarise with the exercise and also to ask questions, as suggested by Pageaux (2016). After familiarisation with the exercise they rested for a minute before testing. Once demonstrating clearly their acquaintance with the instructions, the initial position for variable $V 5$ is assumed (see the Methodology section). Counting time is initiated when the knees are lifted-while the clinician arranges the final kinematic position - and the position is held for a total of fifteen seconds. Just after testing $\mathbf{V}$, participants walk a few steps, and then the initial position for variable $\mathbf{V} \mathbf{6}$ is assumed. During the interval between tests, the participant still remains silent and assumes the initial position. Thus, the participant rests in position for thirty seconds or more if needed. After resting, instruction is then given to lift the knees-arranging the final position as before- and this position is held for a total of fifteen seconds. The score is given just after testing the perceived sensation of effort on both sides. In case of problems in assuming the position during the test, the participant is invited to stand up and walk a few steps, and then is again demonstrated how to assume the position. In other words, if a problem occurs during performance of one of the tests, the participant is invited first to walk and then to sit down resting while the demonstration is repeated. These variables are tested in sequence and participants must have knowledge about the procedure and of course it should be clear what is needed to score.

In Groups 2 and 3, participants first received instruction on how to score perceived effort on a classical Borg scale from six to twenty. Other instructions were delivered in the same way as for Group 1.

5) Criteria adopted to calculate the interstimulus interval (ISI) from video records. The ISI was analysed by a mechanical engineer blinded to the study using Kinovea (Kinovea is open source software and proved to be reliable and valid (Puig-Diví et al., 2017)). Mean values (of seven subjects from Group B) are presented here; the error assumed is $20 \mathrm{~ms}$. The amount of time that it takes to transfer the tensile force to the participant's hand ranges from a minimum of $100 \mathrm{~ms}$ to a maximum of $1.480 \mathrm{~ms}$ (mean, $710 \mathrm{~ms}$ ). Once the tensile force is transferred, the participant reacts to control the acceleration of his hand. The time for initiating the reaction varies from a minimum of $150 \mathrm{~ms}$ to a maximum of $340 \mathrm{~ms}$ (mean, $230 \mathrm{~ms}$ ). Finally, the amount of time that the participant maintains his hand stabilised in position A, i.e. the dwell time, ranges between $300 \mathrm{~ms}$ and $4.120 \mathrm{~ms}$ (mean, $1.490 \mathrm{~ms}$ ). Five phases can be distinguished, which are easily observed in a slow-motion video, as follows. The main events are the action of the tensile force acting on the participant's hand, and the reaction of 
the participant to move the hand back to the specified coordinate, position A. The chronology, which is given below, was used to calculate the time it takes to transfer the tensile force to the participant's hand, the reaction time, and the dwell time in position A. In phase 1 (release start), the clinician starts to release the tensile force, that is, the elastic tubing is partially transferred to the participant's hand. In phase 2 (release end), the clinician finishes releasing the elastic tubing, that is, the tensile force is completely transferred to the participant's hand. In phase 3 (hand moves), the participant's hand gains significant speed compared to phase 1 . In phase 4 (hand reacts), the participant reacts to control the hand's position. In phase 5 (hand stabilises), the participant stabilises his hand in position A for a dwell time. In this phase 5 , it is still possible to observe some small oscillations in the hand's position during the dwell time, but these oscillations are significantly lower in amplitude compared to those in phase 3.

\begin{tabular}{|c|c|}
\hline 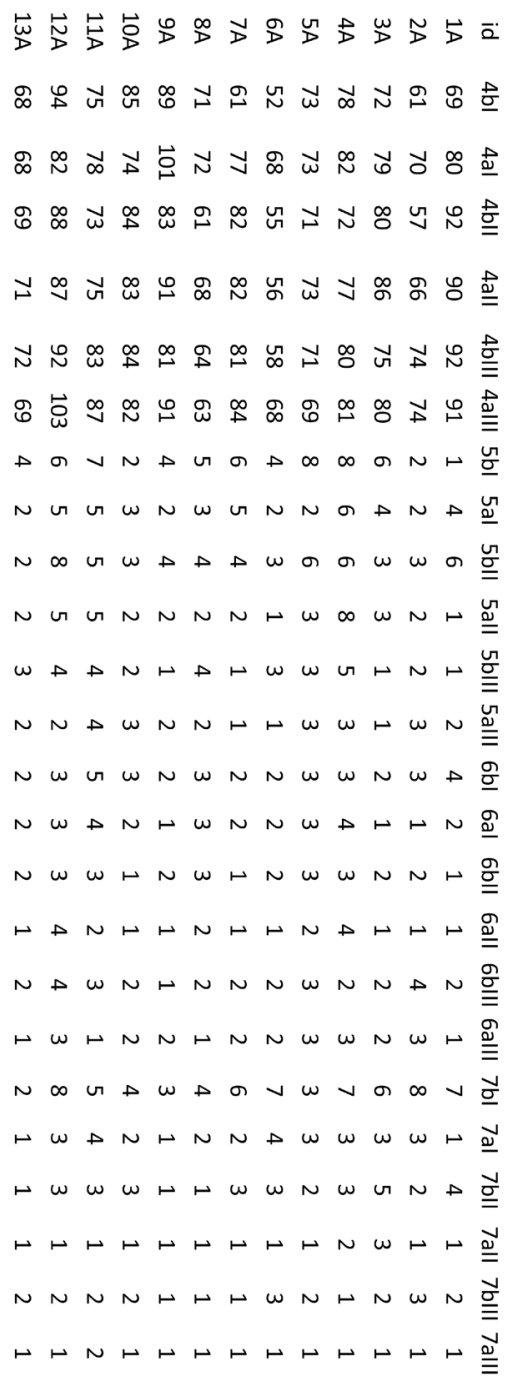 & 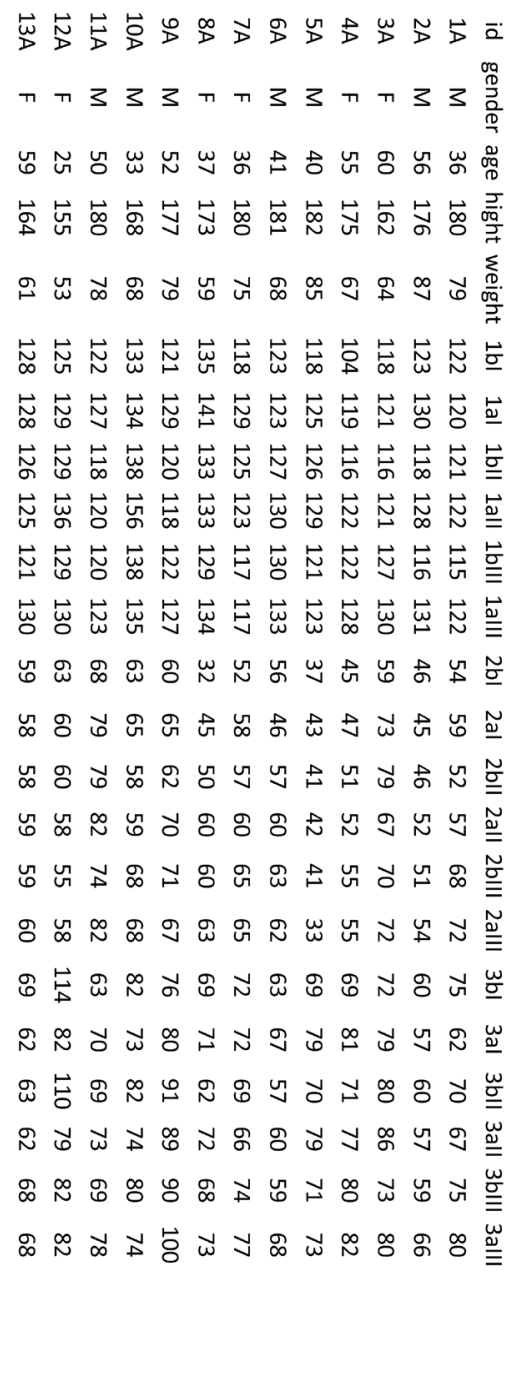 \\
\hline
\end{tabular}


id gender age hight weight $1 \mathrm{bl}$ 1al 2bl 2al 3bl 3al 4bl 4al 5bl 5al 6bl 6al 7bl 7al

$\begin{array}{lllllllllllllllllll}1 B & M & 54 & 187 & 90 & 126 & 123 & 50 & 45 & 74 & 73 & 62 & 73 & 13 & 13 & 15 & 12 & 4 & 2\end{array}$

$\begin{array}{lllllllllllllllllll}2 B & M & 46 & 196 & 113 & 114 & 119 & 62 & 69 & 75 & 72 & 60 & 72 & 12 & 11 & 13 & 10 & 5 & 3\end{array}$

$\begin{array}{lllllllllllllllllll}3 B & M & 27 & 173 & 72 & 121 & 121 & 62 & 62 & 72 & 72 & 71 & 68 & 13 & 13 & 13 & 13 & 0 & 0\end{array}$

$\begin{array}{lllllllllllllllllll}4 \mathrm{~B} & \mathrm{M} & 28 & 172 & 81 & 131 & 136 & 57 & 57 & 90 & 84 & 83 & 83 & 9 & 6 & 11 & 6 & 1 & 0\end{array}$

$\begin{array}{lllllllllllllllllll}5 \mathrm{~B} & \mathrm{M} & 22 & 191 & 79 & 126 & 128 & 65 & 72 & 90 & 81 & 86 & 81 & 12 & 11 & 10 & 9 & 3 & 0\end{array}$

$\begin{array}{lllllllllllllllllll}6 B & M & 56 & 172 & 74 & 122 & 125 & 61 & 65 & 72 & 80 & 65 & 80 & 9 & 11 & 15 & 9 & 2 & 1\end{array}$

$\begin{array}{lllllllllllllllllll}7 \mathrm{~B} & \mathrm{M} & 27 & 195 & 75 & 117 & 119 & 74 & 73 & 67 & 70 & 53 & 62 & 12 & 9 & 11 & 8 & 2 & 2\end{array}$

$\begin{array}{lllllllllllllllllll}8 \mathrm{~B} & \mathrm{M} & 52 & 183 & 96 & 110 & 118 & 62 & 65 & 65 & 62 & 52 & 63 & 15 & 12 & 13 & 9 & 2,5 & 0,5\end{array}$

$\begin{array}{lllllllllllllllllll}9 \mathrm{~B} & \mathrm{M} & 45 & 178 & 79 & 125 & 124 & 58 & 49 & 72 & 72 & 62 & 72 & 9 & 11 & 12 & 12 & 3 & 0,5\end{array}$

$\begin{array}{lllllllllllllllllll}10 B & M & 33 & 178 & 80 & 126 & 126 & 44 & 47 & 62 & 68 & 53 & 68 & 13 & 12 & 11 & 12 & 3 & 1,5\end{array}$

$\begin{array}{lllllllllllllllllll}11 \mathrm{~B} & \mathrm{~F} & 30 & 159 & 55 & 119 & 120 & 63 & 63 & 64 & 72 & 56 & 72 & 15 & 11 & 13 & 11 & 6 & 2\end{array}$

$\begin{array}{lllllllllllllllllll}12 B & F & 40 & 165 & 57 & 116 & 116 & 57 & 65 & 52 & 56 & 62 & 56 & 13 & 9 & 11 & 12 & 2 & 1\end{array}$

$\begin{array}{lllllllllllllllllll}13 B & F & 47 & 165 & 54 & 123 & 123 & 58 & 61 & 61 & 58 & 74 & 61 & 8 & 7 & 7 & 7 & 0 & 0\end{array}$

$\begin{array}{lllllllllllllllllll}1 C & M & 48 & 180 & 80 & 119 & 115 & 56 & 67 & 63 & 65 & 58 & 65 & 12 & 12 & 9 & 9 & 0 & 0\end{array}$

$\begin{array}{lllllllllllllllllll}2 \mathrm{C} & \mathrm{M} & 33 & 170 & 70 & 136 & 142 & 56 & 60 & 65 & 72 & 63 & 71 & 9 & 8 & 9 & 7 & 4 & 3\end{array}$

$\begin{array}{lllllllllllllllllll}3 \mathrm{C} & \mathrm{F} & 22 & 162 & 56 & 113 & 121 & 60 & 55 & 71 & 70 & 64 & 70 & 10 & 9 & 8 & 9 & 1 & 0,3\end{array}$

$\begin{array}{lllllllllllllllllll}4 \mathrm{C} & \mathrm{M} & 52 & 177 & 77 & 115 & 118 & 61 & 59 & 76 & 72 & 69 & 72 & 6 & 8 & 7 & 6 & 0,3 & 0\end{array}$

$\begin{array}{lllllllllllllllllll}5 \mathrm{C} & \mathrm{F} & 22 & 176 & 67 & 128 & 130 & 38 & 58 & 69 & 69 & 74 & 79 & 13 & 14 & 12 & 13 & 1 & 0\end{array}$

$\begin{array}{lllllllllllllllllll}6 \mathrm{C} & \mathrm{M} & 35 & 193 & 93 & 114 & 111 & 61 & 57 & 63 & 71 & 68 & 72 & 11 & 9 & 13 & 9 & 3 & 0,5\end{array}$

$\begin{array}{lllllllllllllllllll}7 C & M & 33 & 178 & 80 & 109 & 111 & 52 & 58 & 69 & 68 & 61 & 67 & 12 & 10 & 11 & 10 & 3 & 0\end{array}$

$\begin{array}{lllllllllllllllllll}8 \mathrm{C} & \mathrm{M} & 26 & 185 & 99 & 113 & 116 & 56 & 50 & 56 & 59 & 63 & 61 & 13 & 11 & 9 & 9 & 2 & 0,5\end{array}$

$\begin{array}{lllllllllllllllllll}9 \mathrm{C} & \mathrm{M} & 38 & 183 & 75 & 126 & 126 & 64 & 64 & 62 & 67 & 73 & 66 & 8 & 9 & 7 & 8 & 1 & 0,3\end{array}$

$\begin{array}{lllllllllllllllllll}10 \mathrm{C} & \mathrm{M} & 35 & 183 & 90 & 125 & 125 & 61 & 59 & 64 & 67 & 57 & 65 & 11 & 12 & 13 & 14 & 1 & 0\end{array}$

$\begin{array}{lllllllllllllllllll}11 C & M & 46 & 178 & 77 & 104 & 106 & 62 & 57 & 73 & 63 & 62 & 63 & 11 & 10 & 10 & 10 & 0,3 & 0\end{array}$

$\begin{array}{lllllllllllllllllll}12 \mathrm{C} & \mathrm{F} & 53 & 153 & 66 & 111 & 111 & 49 & 48 & 70 & 73 & 68 & 73 & 15 & 12 & 12 & 12 & 1 & 0\end{array}$

$\begin{array}{lllllllllllllllllll}13 \mathrm{C} & \mathrm{M} & 30 & 179 & 80 & 130 & 127 & 57 & 57 & 60 & 57 & 56 & 57 & 11 & 11 & 12 & 12 & 2 & 1\end{array}$ 